\title{
FANO COMPACTIFICATIONS OF CONTRACTIBLE AFFINE 3-FOLDS WITH TRIVIAL LOG CANONICAL DIVISORS
}

\author{
MASARU NAGAOKA
}

\begin{abstract}
T. Kishimoto raised the problem to classify all compactifications of contractible affine 3-folds into smooth Fano 3-folds with second Betti number two and classified such compactifications whose $\log$ canonical divisors are not nef. In this article, we show that there are 14 deformation equivalence classes of smooth Fano 3-folds which can admit structures of such compactifications whose log canonical divisors are trivial. We also construct an example of such compactifications with trivial log canonical divisors for each of all the 14 classes.
\end{abstract}

\section{Contents}

1. Introduction.

2. Preliminaries

2.1. Gorenstein del Pezzo surfaces

2.2. Topologies of varieties

3. Boundary divisors

4. Construction of examples of type (A)

4.1. Affine modifications

4.2. The types (A1) and (A2)

4.3. The type (A3)

4.4. The type (A4)

4.5. Another example of type (A3)

5. Construction of examples of type (B)

5.1. The characterization of $\mathbb{A}^{3}$

5.2. The type (B3)

5.3. The types (B1) and (B2)

6. Exclusion of imprimitive Fano 3-folds

6.1. The image of contraction $\varphi_{1}: V \rightarrow W_{1}$

6.2. On the conditions in Lemma 4.3

6.3. The case $W_{1}=\mathbb{P}^{3}$

6.4. The case $W_{1}=\mathbb{Q}^{3}$

6.5. The case $W_{1}=V_{5}$

7. Exclusion of primitive Fano 3-folds

References

2010 Mathematics Subject Classification. Primary: 14R10; Secondary: 14J45, 14J30.

Key words and phrases. Contractible affine threefolds, compactifications, Fano threefolds. 


\section{INTRODUCTION.}

Throughout the present article we work over the field of complex numbers $\mathbb{C}$. In Hir54, F. Hirzebruch raised the problem to classify all compactifications of the affine space $\mathbb{A}^{n}$ into compact complex manifolds with second Betti number $B_{2}=1$ for all $n$. By contributions of M. Furushima, N. Nakayama, Th. Peternell and M. Schneider (cf. Fur86, Fur90, Fur92, Fur93, F-N89a, F-N89b, Pet89, P-S88]), the classification of compactifications of $\mathbb{A}^{3}$ into smooth projective 3 -folds with $B_{2}=1$ was completed.

Let $V$ be a smooth projective 3 -fold with $B_{2}=1$ which contains $\mathbb{A}^{3}$ as an open set. Let $D:=V \backslash \mathbb{A}^{3}$. Then $V$ is a Fano 3-fold and the anticanonical divisor $-K_{V}$ is linearly equivalent to $r D$, where $r$ is the Fano index of $V$. First, in [Fur86, F-N89a, F-N89b, P-S88, the pairs $(V, D)$ with $r \geq 2$ were classified. Then Furushima [Fur93] classified the pairs $(V, D)$ with $r=1$.

T. Kishimoto Kis05 observed that their arguments make use of only the contractibility of $\mathbb{A}^{3}$ and that, by the arguments, it is possible to classify compactifications of contractible affine 3-folds into smooth Fano 3-folds with $B_{2}=1$. After that, Kishimoto raised the following problem as the corresponding problem in the case when $B_{2}=2$.

Problem 1.1. Classify triplets $\left(V, D_{1} \cup D_{2}, U\right)$, where $V$ is a smooth Fano 3-fold with $B_{2}=2, D_{1}$ and $D_{2}$ are prime divisors on $V$, and $U$ is the complement $V \backslash\left(D_{1} \cup D_{2}\right)$ which is a contractible affine 3-fold.

We often call $D_{1}$ and $D_{2}$ the boundary divisors and $K_{V}+D_{1}+D_{2}$ the log canonical divisor.

Using the list [M-M81, Table 2], Kishimoto classified all such triplets in [Kis05] when the log canonical divisors are not nef. For this reason, we consider such triplets whose log canonical divisors are nef. Then, by virtue of the Kawamata-Shokurov base point free theorem, the log canonical divisor for such a triplet is semiample and we denote by $\kappa\left(K_{V}+D_{1}+D_{2}\right)$ the dimension of the image of the morphism given by the linear system $\left|m\left(K_{V}+D_{1}+D_{2}\right)\right|$ for sufficiently large $m$.

In this article, we shall investigate triplets $\left(V, D_{1} \cup D_{2}, U\right)$ as in Problem 1.1 satisfying the following condition:

$$
K_{V}+D_{1}+D_{2} \text { is nef and } \kappa\left(K_{V}+D_{1}+D_{2}\right)=0 .
$$

If the condition holds for a triplet $\left(V, D_{1} \cup D_{2}, U\right)$, then $m\left(K_{V}+D_{1}+D_{2}\right)=0$ for sufficiently large $m$. Since the Picard groups of $V$ is torsion-free, we have $m=1$. Hence the condition is rewritten as

$$
(\dagger): K_{V}+D_{1}+D_{2}=0 \text {. }
$$

In this article, a triplet $\left(V, D_{1} \cup D_{2}, U\right)$ is called a triplet with ( $\left.\dagger\right)$ if it is as in Problem 1.1 and satisfies $(\dagger)$. Note that a similar condition $K_{V}+D=0$ holds for every smooth projective compactification $(V, D)$ of $\mathbb{A}^{3}$ with $B_{2}=1$ and $r=1$.

Our main result is the following, where we denote by $\mathbb{Q}^{3}$ the smooth quadric 3 -fold and by $V_{5}$ the smooth quintic del Pezzo 3 -fold.

\section{Theorem 1.1.}


(1) Let $\left(V, D_{1} \cup D_{2}, U\right)$ be a triplet as in Problem 1.1. Suppose the triplet satisfies $(\dagger)$. Then there are only 14 deformation equivalence classes to one of which the Fano 3-fold $V$ belongs. We give a precise description of $V$ below.

(A) If $V$ is imprimitive, then it is the blow-up of a Fano 3-fold $W$ along a smooth curve $C$ such that one of the following holds:

(A1) $W \cong \mathbb{P}^{3}$ and $C$ is an elliptic curve of degree 3,4 .

(A2) $W \cong \mathbb{P}^{3}$ and $C$ is a rational curve of degree $1,2,3,4$.

(A3) $W \cong \mathbb{Q}^{3}$ and $C$ is a rational curve of degree $1,2,3,4$.

(A4) $W \cong V_{5}$ and $C$ is a rational curve of degree $1,2,3$.

(B) If $V$ is primitive, then $V$ is one of the following:

(B1) $V \subset \mathbb{P}^{2} \times \mathbb{P}^{2}$ is a divisor of bidegree $(1,2)$.

(B2) $V \cong \mathbb{P}^{1} \times \mathbb{P}^{2}$.

(B3) $V \cong \mathbb{P}_{\mathbb{P}^{2}}(\mathcal{O} \oplus \mathcal{O}(2))$, which is the blow-up of the cone over the Veronese surface at the vertex.

(2) For each $\mathcal{C}$ of 14 classes as in (1), there is a triplet $\left(V, D_{1} \cup D_{2}, U\right)$ as in Problem 1.1 satisfying $(\dagger)$ such that $V$ belongs to $\mathcal{C}$.

Remark 1.2. To prove Theorem 1.1, we use the list [M-M81, Table 2] of smooth Fano 3 -folds with $B_{2}=2$. Each type as in Theorem 1.1 corresponds to the numbers in [ibid.] as follows:

(A1) $V$ is of No.25 or No.28.

(A2) $V$ is of No.22, No.27, No.30 or No.33.

(A3) $V$ is of No.21, No.26, No.29 or No.31.

(A4) $V$ is of No.20, No.22 or No.26.

(B1) $V$ is of No.24.

(B2) $V$ is of No.34.

(B3) $V$ is of No.36.

Note that No.22 and No.26 appear twice above.

In this article, a triplet $\left(V, D_{1} \cup D_{2}, U\right)$ with $(\dagger)$ is called of type $(*)$ if $V$ belongs to the type $(*)$ of Theorem 1.1 .

Theorem 1.1 determines the candidate of the ambient spaces $V$ of triplets $\left(V, D_{1} \cup D_{2}, U\right)$ with $(\dagger)$. In the forthcoming article [Nag2, we determine all triplets $\left(V, D_{1} \cup D_{2}, U\right)$ with ( $\dagger$ ) of type (A1) or (A2) and it turns out that $U \cong \mathbb{A}^{3}$ except for one case.

This paper is structured as follows.

In 92 , we recall some facts about Gorenstein del Pezzo surfaces (\$2.1) and topologies of varieties (\$2.2), which we use to prove Theorem 1.1.

In 93 , we show that the linear equivalence classes of the boundary divisors of a triplet with $(\dagger)$ are uniquely determined up to permutation of the boundary divisors. In Lemma 3.5, we also prove an important equality between topological invariants about a triplet with $(\dagger)$, which is a key to prove Theorem 1.1 (1).

In 84 , we construct an examples of triplets with $(\dagger)$ for each of all deformation equivalence classes of ambient spaces of type (A). To do so, we introduce a sufficient condition of triplets with $(\dagger)$ of type (A) in Lemma 4.3. We actually construct examples in 4.24.4. We also show that the above condition is not a necessary condition in $\$ 4.5$. 
In 95 , we construct an examples of triplets with $(\dagger)$ for each of remaining classes, i.e. of type (B). We review Kaliman's characterization of $\mathbb{A}^{3}$ (Theorem 5.11) in $\$ 5.1$ to construct examples explicitly in $\$ 5.2-\$ 5.3$. By combining $\$ 4$ and $\$$, we complete the proof of Theorem $1.1(2)$.

In 96 7, we prove Theorem 1.1 (1) by showing that, unless the ambient space is of type (A) and (B), a triplet with ( $\dagger$ ) does not satisfy the equality of Lemma 3.5. 96 (resp. 97) deals with the case that the ambient space is imprimitive (resp. primitive).

Notation and Conventions. In this article, we always assume that Fano varieties are smooth. We use the numbers assigned to Fano 3 -folds with $B_{2}=2$ as in [M-M81, Table 2]. We also employ the following notation.

- $B_{i}(X)$ : the $i$-th Betti number of a topological space $X$.

- $\chi_{\text {top }}(X)$ : the topological Euler number of a topological space $X$.

- Sing $X$ : the singular locus of a variety $X$.

- $K_{X}$ : the canonical divisor of a Gorenstein variety $X$.

- $p_{a}(r)$ : the arithmetic genus of a curve $r$.

- $E_{f}$ : the exceptional divisor of a birational morphism $f$.

- $f_{*}^{-1}(D)$ : the strict transform of a divisor $D$ by a birational map $f$.

- $\mathbb{Q}^{3}$ : the smooth quadric 3-fold in $\mathbb{P}^{4}$.

- $V_{d}$ : a smooth del Pezzo 3-fold of degree $d$.

- $\mathbb{Q}_{0}^{2}$ : the quadric cone in $\mathbb{P}^{3}$.

- $\mathbb{F}_{n}$ : the Hirzebruch surface of degree $n$.

- $f_{n}$ : a ruling of $\mathbb{F}_{n}$.

- $\Sigma_{n}$ : the minimal section of $\mathbb{F}_{n}$.

Acknowledgement. The author is greatly indebted to Prof. Hiromichi Takagi, his supervisor, for his encouragement, comments, and suggestions. He also wishes to express his gratitude to Prof. Takashi Kishimoto for his helpful comments and suggestions. He also would like to express his gratitude to Dr. Akihiro Kanemitsu and Dr. Takeru Fukuoka for giving very significant advice in order to make this article more readable.

\section{Preliminaries}

2.1. Gorenstein del Pezzo surfaces. In this subsection, we review some facts on Gorenstein del Pezzo surfaces.

Theorem 2.1 (cf.[H-W81], A-F83]). Let $S$ be a Gorenstein del Pezzo surface of degree $d \geq 3$.

(1) If $S$ is normal and rational, then $\chi_{\mathrm{top}}(S)=2+B_{2}(S) \geq 3$.

(2) If $S$ is normal and irrational, then $\chi_{\text {top }}(S)=1$.

(3) If $S$ is non-normal, then $\chi_{\text {top }}(S) \geq 2$.

(4) If $S$ is not a cone over a curve, then $\chi_{\mathrm{top}}(S) \geq 3$.

Lemma 2.2. Let $S$ be a Gorenstein del Pezzo surface of degree $d \geq 4$ which is the cone over a curve. Then $S$ cannot be embedded in smooth 3-fold.

Proof. The surface $S \subset \mathbb{P}^{d}$ is an intersection of quadric hypersurfaces by Fuj90, Corollary 1.5]. Since $S$ is the cone over a curve, such quadric hypersurface is singular at the vertex of $S$. Then we can compute that the 
embedding dimension of $S$ at the vertex is $d \geq 4$. Hence we have the assertion.

Notation 1. For a non-normal surface $S$, we use the following notation:

- $\sigma_{S}: \bar{S} \rightarrow S:$ the normalization.

- $\mathcal{C}_{S} \subset \mathcal{O}_{S}$ : the conductor ideal of $\sigma_{S}$.

- $E_{S}:=V_{S}\left(\mathcal{C}_{S}\right)$ and $\bar{E}_{S}:=V_{\bar{S}}\left(\sigma_{S}^{*}\left(\mathcal{C}_{S}\right)\right)$ : the subschemes defined by $\mathcal{C}_{S}$. We call $E_{S}\left(\operatorname{resp} . \bar{E}_{S}\right)$ the conductor locus of $S(\operatorname{resp} . \bar{S})$.

The following lemma is used in our proof of Propositions 6.9 and 6.14.

Lemma 2.3. Let $S$ be a non-normal Gorenstein del Pezzo surface of degree $d \geq 3$. Suppose that $S$ belongs to the class $(\mathrm{C})$ in $\left[\mathrm{A}-\mathrm{F} 83\right.$ and $\bar{E}_{S}$ is reducible. Then each member $D \in\left|-K_{S}\right|$ satisfies $B_{2}(D)<d$.

Proof. Since $S$ belongs to the class (C) of A-F83, its normalization $\bar{S}$ is isomorphic to $\mathbb{F}_{d-2}$ and the conductor locus $\bar{E}_{S}$ of $\bar{S}$ is linearly equivalent to $\Sigma_{d-2}+f_{d-2}$. Since $\bar{E}_{S}$ is reducible, it consists of $\Sigma_{d-2}$ and a ruling $F$ of $\mathbb{F}_{d-2}$. The normalization $\sigma_{S}: \bar{S} \rightarrow S$ induces isomorphisms from both $\Sigma_{d-2}$ and $F$ to $E_{S}$. We also have $-\sigma_{S}^{*}\left(K_{S}\right) \sim \Sigma_{d-2}+(d-1) f_{d-2}$.

As $B_{2}(D) \leq\left(D \cdot-K_{S}\right)=d$, it suffices to show that $B_{2}(D) \neq d$. On the contrary, suppose that $B_{2}(D)=d$. Then $D$ consists of curves $r_{1}, \ldots, r_{d}$ with $\left(r_{i} \cdot-K_{S}\right)=1$ for $i=1, \ldots, d$.

Fix $i \in\{1, \ldots, d\}$. Then $r_{i} \not \subset E_{S}$ since $D$ is Cartier. As $\bar{E}_{S}=\Sigma_{d-2}+F$, we have $\sigma_{S_{*}}^{-1}\left(r_{i}\right) \neq \Sigma_{d-2}$ or $F$. If we write $\sigma_{S_{*}}^{-1}\left(r_{i}\right) \sim a \Sigma_{d-2}+b f_{d-2}$, then it holds that $1=\left(r_{i} \cdot-K_{S}\right)_{S}=\left(\sigma_{S *}^{-1}\left(r_{i}\right) \cdot-\sigma_{S}^{*} K_{S}\right)_{S}=\left(a \Sigma_{d-2}+b f_{d-2} \cdot \Sigma_{d-2}+\right.$ $\left.(d-1) f_{d-2}\right)_{\bar{S}}=a+b$. Hence $\sigma_{S *}^{-1}\left(r_{i}\right) \sim f_{d-2}$ for $i=1, \ldots, d$. However this implies that $D$ is disconnected since $\sigma_{S}$ is as stated, which contradicts the ampleness of $D$.

2.2. Topologies of varieties. In this subsection, we summarize some facts about the topologies of varieties which we apply in our proof of Propositions 6.6 and 6.7 .

Lemma 2.4. Let $X$ be a smooth contractible affine 3-fold, $S \subset X$ a closed irreducible normal surface and $r \subset S$ a closed irreducible smooth curve. Let $\varphi: \mathrm{Bl}_{r} X \rightarrow X$ be the blow-up of $X$ along $r$. Suppose that $N:=\mathrm{Bl}_{r} X \backslash \varphi_{*}^{-1}(S)$ is contractible. Then we have

$$
H_{i}(S \backslash \operatorname{Sing} S, \mathbb{Z})= \begin{cases}\mathbb{Z} & i=0 \\ H_{1}(r \backslash(r \cap \operatorname{Sing} S), \mathbb{Z}) & i=1 \\ \mathbb{Z}^{\sharp \operatorname{Sing} S} & i=3 \\ 0 & \text { otherwise. }\end{cases}
$$

Proof. Let $M:=X \backslash \operatorname{Sing} S, S^{\prime}:=S \backslash \operatorname{Sing} S$ and $E:=E_{\varphi} \cap N$. Note that $N=\left(\mathrm{Bl}_{r \backslash(r \cap \operatorname{Sing} S)} M\right) \backslash \varphi_{*}^{-1}\left(S^{\prime}\right)$. Applying the Thom isomorphism to the pair $(X$, Sing $S)$, we get the following exact sequence:

$$
\cdots \rightarrow H_{i-5}(\operatorname{Sing} S, \mathbb{Z}) \rightarrow H_{i}(M, \mathbb{Z}) \rightarrow H_{i}(X, \mathbb{Z}) \rightarrow H_{i-6}(\operatorname{Sing} S, \mathbb{Z}) \rightarrow \cdots
$$

Note that $N$ is contractible by the assumption and $E$ is an $\mathbb{A}^{1}$-bundle over $r \backslash(r \cap \operatorname{Sing} S)$. Thus we can calculate the singular homologies of $M, N$, 
and $E$. Then we have

$$
\begin{aligned}
& H_{i}(M, \mathbb{Z})= \begin{cases}\mathbb{Z} & i=0 \\
\mathbb{Z}^{\sharp \operatorname{Sing} S} & i=5 \\
0 & i \text { : otherwise, }\end{cases} \\
& H_{i}(E, \mathbb{Z})= \begin{cases}\mathbb{Z} & H_{i}(N, \mathbb{Z})= \begin{cases}\mathbb{Z} & i=0 \\
0 & i \text { : otherwise, }\end{cases} \\
H_{1}(r \backslash(r \cap \operatorname{Sing} S), \mathbb{Z}) & i=1 \\
0 & i \text { : otherwise. }\end{cases}
\end{aligned}
$$

As $\varphi\left\lceil_{N \backslash E}: N \backslash E \rightarrow M \backslash S^{\prime}\right.$ is an isomorphism, the Thom isomorphism to the pairs $\left(M, S^{\prime}\right)$ and $(N, E)$ gives us the following commutative diagram with the exact rows:

$$
\begin{aligned}
& \cdots \rightarrow H_{i-1}(E, \mathbb{Z}) \longrightarrow H_{i}(N \backslash E, \mathbb{Z}) \longrightarrow H_{i}(N, \mathbb{Z}) \rightarrow H_{i-2}(E, \mathbb{Z}) \longrightarrow \cdots
\end{aligned}
$$

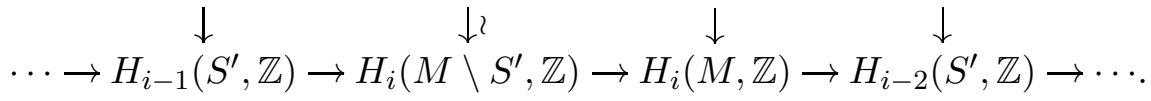

Substituting the terms of the commutative diagram by $(*)$, we have the assertion.

Lemma 2.5. Let $f: \widetilde{X} \rightarrow X$ be a proper morphism of projective algebraic varieties such that its restriction $f^{-1}(U) \rightarrow U$ over a dense open subset $U$ is isomorphic. Let $Y:=X \backslash U$ and $\tilde{Y}:=f^{-1}(Y)$, then there exists an exact sequence of cohomologies:

$$
\cdots \rightarrow H^{i}(X, \mathbb{Z}) \rightarrow H^{i}(Y, \mathbb{Z}) \oplus H^{i}(\tilde{X}, \mathbb{Z}) \rightarrow H^{i}(\tilde{Y}, \mathbb{Z}) \rightarrow H^{i+1}(X, \mathbb{Z}) \rightarrow \cdots
$$

Proof. This follows by the same method as in the proof of [sh14, Lemma 8.1.7].

Definition 2.6. For a normal surface singularity $(X, x), \pi_{X, x}:=\lim _{U} \pi_{1}(U \backslash$ $\{x\})$ is called the local fundamental group of $(X, x)$. We say that $\pi_{X, x}$ is perfect if its abelianization is trivial.

Theorem 2.7 (cf. [Bri68, Satz 2.8]). Let $(X, x)$ be a normal surface singularity. Then the following are equivalent:

(1) $(X, x)$ is a quotient singularity.

(2) The local fundamental group $\pi_{X, x}$ is finite.

Theorem 2.8 (cf. [Bri68, [B-D89, Theorem1.4(a)]). Let $(X, x)$ be a rational double point. Then the following are equivalent:

(1) $(X, x)$ is an $E_{8}$-singularity.

(2) The local fundamental group $\pi_{X, x}$ is perfect.

Theorem 2.9 (cf. [KPR89]). Let $G$ be a reductive algebraic group acting algebraically on a contractible affine variety $X$. Then the algebraic quotient $X / / G$ is also contractible.

\section{BOUNDARY DIVISORS}

In this section, we study properties of the boundary divisors of triplets with $(\dagger)$. 
Notation 2. For a Fano 3-fold $V$ with $B_{2}=2$, we use the following notation for $i=1,2$ :

- $\varphi_{i}$ : the extremal contractions of $V$.

- $W_{i}$ : the image of $\varphi_{i}$.

- $H_{i}$ : the pullback of the ample generator of Pic $W_{i}$.

- $\mu_{i}$ : the length of $\varphi_{i}$, i.e. $\mu_{i}:=\min \left\{\left(-K_{V} \cdot l\right) \in \mathbb{N} \mid l\right.$ : a curve such that $\varphi_{i}(l)$ is a point $\}$.

- $l_{i} \subset V$ : a curve such that $\left(-K_{V} \cdot l_{i}\right)=\mu_{i}$ and $\varphi_{i}(l)$ is a point.

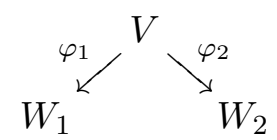

Theorem 3.1 (cf. [M-M83, Theorem 5.1]). With Notation 2, we have the following:

(1) Pic $V=\mathbb{Z}\left[H_{1}\right] \oplus \mathbb{Z}\left[H_{2}\right]$.

(2) $-K_{V} \sim \mu_{1} H_{2}+\mu_{2} H_{1}$.

(3) $\left(H_{i} \cdot l_{j}\right)=1-\delta_{i j}$ for all $i, j$, where $\delta_{i j}$ is Kronecker's delta.

In the remaining of this section, we fix a triplet $\left(V, D_{1} \cup D_{2}, U\right)$ with $(\dagger)$.

Lemma 3.2 (cf. Kis05, Lemma.2.1]). It holds that Pic $V=\mathbb{Z}\left[D_{1}\right] \oplus \mathbb{Z}\left[D_{2}\right]$.

Lemma 3.3. Take $m_{i j} \in \mathbb{Z}$ such that $D_{i} \sim m_{i 1} H_{1}+m_{i 2} H_{2}$. Then $m_{i j} \geq 0$ for all $i, j$.

Proof. By symmetry, we have only to show $m_{11} \geq 0$. Suppose that $m_{11}<0$. Then $m_{12}>0$ since $D_{1}$ is a non-zero effective divisor. By the condition $(\dagger)$ and Theorem 3.1 (3), we have $m_{11}+m_{21} \geq 1$ and $m_{12}+m_{22} \geq 1$. Hence we have

$$
\begin{aligned}
m_{11} m_{22}-m_{12} m_{21} & \leq m_{11} m_{22}-m_{12}\left(-m_{11}+1\right)=m_{11}\left(m_{22}+m_{12}\right)-m_{12} \\
& \leq m_{11}-m_{12} \\
& \leq-2,
\end{aligned}
$$

which implies that the matrix $\left(m_{i j}\right)_{1 \leq i, j \leq 2}$ is not unimodular. This contradicts to Theorem 3.1 (1) and Lemma 3.2.

Proposition 3.4. We have the following:

(1) For every $k \in\{1,2\}$ such that $\mu_{k}=1$, we have $H_{k} \sim D_{l}$ for some $l$.

(2) If $\mu_{1}, \mu_{2} \geq 2$, then $V \cong \mathbb{P}^{1} \times \mathbb{P}^{2}$.

Proof. Let us define $m_{i j}$ as in Lemma 3.3. Note that the matrix $\left(m_{i j}\right)_{1 \leq i, j \leq 2}$ is unimodular by Lemma 3.2 and Theorem 3.1 (1). We have $\mu_{i}=m_{1 i}+m_{2 i}$ for $i=1,2$ by the condition $(\dagger)$.

(1) We may assume that $k=1$. Then $\left(m_{12}, m_{22}\right)=(1,0),(0,1)$ by Lemma 3.3. If the former holds, then $\operatorname{det}\left(m_{i j}\right)=-m_{21}=-1$ and hence $H_{1} \sim D_{2}$. If the latter holds, then $H_{1} \sim D_{1}$ by the same method.

(2) If $\left(\mu_{1}, \mu_{2}\right) \neq(2,2)$ in addition, then $V \cong \mathbb{P}^{1} \times \mathbb{P}^{2}$ by M-M83. Suppose that $\left(\mu_{1}, \mu_{2}\right)=(2,2)$. Then $\left(m_{1 k}, m_{2 k}\right)=(2,0),(0,2)$ or $(1,1)$ for $k=1,2$. If one of the former two cases occurs, then $\operatorname{det}\left(m_{i j}\right)$ is even, a contradiction. Hence $m_{i j}=1$ for all $i, j$, which implies $\operatorname{det}\left(m_{i j}\right)=0$, a contradiction.

The following lemma is useful. 
Lemma 3.5. It holds that $\chi_{\text {top }}\left(D_{1} \cap D_{2}\right)=\chi_{\text {top }}\left(D_{1}\right)+\chi_{\text {top }}\left(D_{2}\right)+B_{3}(V)-5$.

Proof. By the Mayer-Vietoris exact sequence, we have $\chi_{\text {top }}\left(D_{1} \cap D_{2}\right)=$ $\chi_{\text {top }}\left(D_{1}\right)+\chi_{\text {top }}\left(D_{2}\right)-\chi_{\text {top }}\left(D_{1} \cup D_{2}\right)$. Since $U$ is contractible, we have $\chi_{\text {top }}\left(D_{1} \cup D_{2}\right)=\chi_{\text {top }}(V)-1=5-B_{3}(V)$.

\section{Construction of examples of type (A)}

In this section, we construct examples of triplets with ( $\dagger$ ) of type (A1)(A4).

4.1. Affine modifications. We review results about affine modifications, which is a technique to construct new contractible affine varieties from wellknown ones.

Definition 4.1 (cf. K-Z99, Kis05]). Let $R$ be an affine domain and let $Z:=\operatorname{Spec}(R)$. Let $I \subset R$ be an ideal and let $f$ be an element of $I$. We denote by $D:=V_{Z}(f) \supset C:=V_{Z}(I)$ the subschemes in $Z$ defined by $f$ and $I$, respectively. Then the affine modification of $Z$ with a locus $(C \subset D)$ is the affine variety $Z^{\prime}$ with a coordinate ring $R^{\prime}:=\Gamma\left(\mathcal{O}_{Z^{\prime}}\right)=R[I / f]$. It is clear that $R \subset R^{\prime} \subset Q(R)$, where $Q(R)$ is the quotient field of $R$. The canonical inclusion $R \subset R^{\prime}$ induces a birational morphism $\tau: Z^{\prime} \rightarrow Z$. We often call this morphism $\tau$ itself as an affine modification with a locus $(C \subset D)$. If $f$ is not a unit in $R^{\prime}$, it defines a divisor $E:=\operatorname{div}(f)_{Z^{\prime}}$ on $Z^{\prime}$ and we have an isomorphism $\left.\tau\right|_{Z^{\prime} \backslash E}: Z^{\prime} \backslash E \rightarrow Z \backslash D$. Then $E$ is said to be an exceptional divisor of the morphism $\tau$.

Theorem 4.2 (cf. [K-Z99, Theorem 3.1, Corollary 3.1]). Let $Z$ be a contractible affine variety. Let $\tau: Z^{\prime} \rightarrow Z$ be an affine modification with a locus $(C \subset D)$. Let $E$ be the exceptional divisor of $\tau$. Suppose that

(I) $Z$ and $Z^{\prime}$ are complex manifolds and $D$ and $E$ are topological manifolds with finite decompositions into irreducible components $D=$ $\sum_{i=1}^{n} D_{i}$ and $E=\sum_{i=1}^{n} E_{i}$ respectively such that $E_{i}=\tau^{*}\left(D_{i}\right)$ for $i=1, \ldots, n$, and

(II) $\tau\left(E_{i}\right) \not \subset$ Sing $D_{i}$ for $i=1, \ldots, n$.

Then the following are equivalent:

(1) $Z^{\prime}$ is contractible.

(2) $\left(\left.\tau\right|_{E_{i}}\right)_{*}: H_{*}\left(E_{i}, \mathbb{Z}\right) \rightarrow H_{*}\left(D_{i}, \mathbb{Z}\right)$ is an isomorphism for all $i=1, \ldots, n$.

Suppose that a Fano 3-fold $V$ is the blow-up of a variety $W$ along a smooth curve and $W$ contains a contractible affine 3 -fold as an open set. Then we use the following lemma to find contractible affine 3 -folds in $V$, which is a corollary of Theorem 4.2 .

Lemma 4.3. Let $W$ be a smooth projective 3-fold. Let $\left(S_{1}, S_{2}, C\right)$ be a triplet of subvarieties of $W$, where $S_{1}$ and $S_{2}$ are closed surfaces and $C$ is a closed smooth curve. Assume that the following holds:

(I) $C \not \subset S_{1}, C \subset S_{2}$, and $C \not \subset$ Sing $S_{2}$.

(II) $W \backslash S_{1}$ is contractible and affine.

(III) $S_{2} \backslash\left(S_{1} \cap S_{2}\right)$ is smooth.

Let $\varphi: V \rightarrow W$ be the blow-up along $C$. Then the following are equivalent: 
(1) the open subvariety $U:=V \backslash\left(\varphi_{*}^{-1}\left(S_{1}\right) \cup \varphi_{*}^{-1}\left(S_{2}\right)\right)$ is contractible and affine.

(2) the inclusion $\iota: C \backslash\left(S_{1} \cap C\right) \hookrightarrow S_{2} \backslash\left(S_{1} \cap S_{2}\right)$ induces an isomorphism $H_{*}\left(C \backslash\left(S_{1} \cap C\right), \mathbb{Z}\right) \rightarrow H_{*}\left(S_{2} \backslash\left(S_{1} \cap S_{2}\right), \mathbb{Z}\right)$.

Proof. Applying Theorem 4.2 by setting $\tau=\left.\varphi\right|_{U},\left(Z^{\prime}, E\right)=\left(U, E_{\varphi} \cap U\right)$ and $(Z, D)=\left(W \backslash S_{1}, S_{2} \backslash\left(S_{1} \cap S_{2}\right)\right)$, we have the assertion.

In the forthcoming article Nag2, we investigate the isomorphism class of $U$ as above when $V$ is of type (A1) or (A2), that is, when $W \cong \mathbb{P}^{3}$.

Notation 3. For closed surfaces $S_{1}$ and $S_{2}$ in a smooth projective 3-fold $W$, we define a condition (I') as

(I') $S_{1}+S_{2} \sim-K_{W}$.

The following lemma shows us that the condition (I) of Lemma 4.3 is necessary to construct triplets $\left(V, D_{1} \cup D_{2}, U\right)$ with $(\dagger)$ of type $(\mathrm{A})$.

Lemma 4.4. Let $\left(V, D_{1} \cup D_{2}, U\right)$ be a triplet as in Problem 1.1. Assume that $V$ is given by the blow-up $\varphi: V \rightarrow W$ of a Fano variety $W$ along a smooth curve $C$. Let $S_{i}:=\varphi\left(D_{i}\right)$ for $i=1,2$. Then $(\dagger)$ holds for $D_{1}$ and $D_{2}$ if and only if (I) and (I') hold for $S_{1}$ and $S_{2}$ up to permutation.

Proof. The if part is obvious. Suppose that $(\dagger)$ holds for the triplet. Since the length of $\varphi$ is one, we may assume $D_{1} \sim \mathcal{O}_{W}(1)$ and $D_{2} \sim \mathcal{O}_{W}(r-1)-E_{\varphi}$ by Proposition 3.4 and the condition $(\dagger)$, where $r$ is the Fano index of $W$. Hence $S_{1}=\varphi\left(D_{1}\right)$ and $S_{2}=\varphi\left(D_{2}\right)$ satisfy (I) and (I').

Remark 4.5. We use the notation as in Lemma 4.4, In $\$ 6.2$, we prove the following relations among $(\dagger)$ and $(\mathrm{I})-(\mathrm{III})$ of Lemma 4.3.

- Suppose $(\dagger)$ holds. Then (II) holds if and only if $B_{2}\left(S_{1}\right)$ is smallest possible among hyperplane sections of $W$.

- Suppose ( $\dagger$ ) and (II) hold, and $S_{2}$ is normal and rational. Then (III) holds.

Hence (II) holds when $\left(V, D_{1} \cup D_{2}, U\right)$ is a triplet with ( $\dagger$ ) of type (A1) or (A2) since $S_{1} \cong \mathbb{P}^{2}$. In the forthcoming article [Nag2], we show that the second relation still holds without the rationality of $S_{2}$.

Now we choose four varieties $C, S_{1}, S_{2}, W$ such that the pair (W, C) satisfies one of the statements (A1)-(A4) and the triplet $\left(S_{1}, S_{2}, C\right)$ satisfies (I)-(III), (I') and (1) of Lemma 4.3. Then we complete the proof of Theorem 1.1 (2) for all deformation equivalent classes as in (A) of Theorem 1.1 by Lemmas 4.3 and 4.4 in 4.24 .4 .

Note that triplets with $(\dagger)$ of type (A) are not necessarily constructed from triplets of subvarieties which satisfy all the condition of Lemma 4.3 , In fact, we shall construct a triplet with ( $\dagger$ ) of type (A3) from a triplet of subvarieties which does not satisfy the condition (II) in $\$ 4.5$.

4.2. The types (A1) and (A2). We construct triplets $\left(V, D_{1} \cup D_{2}, U\right)$ with $(\dagger)$ of types (A1) and (A2).

Proposition 4.6. There exists a triplet $\left(V, D_{1} \cup D_{2}, U\right)$ with $(\dagger)$ such that $V$ is a blow-up of $\mathbb{P}^{3}$ along an elliptic curve of degree $d$ for $d=3,4$. 
Proof. Let $S_{2}$ be the cone over a plane elliptic curve $e$, and $v$ the vertex of $S_{2}$. Let $\pi: \widetilde{S}_{2} \rightarrow S_{2}$ be the minimal resolution. Then $\widetilde{S}_{2}$ is a $\mathbb{P}^{1}$-bundle over $e$, which corresponds to a decomposable vector bundle of rank 2 and degree 3. Let $C_{0}$ (resp. $f$ ) be the minimal section (resp. a ruling) of $\widetilde{S}_{2}$. On $\widetilde{S}_{2}$, take a smooth member $\widetilde{C}_{d} \in\left|C_{0}+d f\right|$ and let $C_{d}:=\pi\left(\widetilde{C}_{d}\right)$, which is an elliptic curve of degree $d$ for $d=3,4$.

For $d=3$, let $S_{1}$ be a hyperplane which contains $v$. Then the triplet $\left(S_{1}, S_{2}, C_{3}\right)$ satisfies the conditions (II), (III) and (I'). Since the intersection $S_{1} \cap S_{2}$ is a sum of rulings of $S_{2}$, the surface $S_{1}$ does not contain $C_{3}$. Hence the condition (I) holds for the triplet $\left(S_{1}, S_{2}, C_{3}\right)$. It also holds that $S_{2} \backslash$ $\left(S_{1} \cap S_{2}\right)$ is isomorphic to an $\mathbb{A}^{1}$-bundle over $C_{3} \backslash\left(S_{1} \cap C_{3}\right)$. Hence the triplet $\left(S_{1}, S_{2}, C_{3}\right)$ satisfies the condition (1).

For $d=4$, let $\widetilde{l}$ be the ruling of $\widetilde{S}_{2}$ such that $\widetilde{l} \cap \widetilde{C}_{4}=\widetilde{l} \cap C_{0}$, and $l:=\pi(\widetilde{l})$. Let $S_{1}$ be a hypersurface which contains $l$. Then $S_{1}$ contains $v$ and hence the triplet $\left(S_{1}, S_{2}, C_{4}\right)$ satisfies the conditions (I)-(III) and (I') for the same reason as the case that $d=3$. By the choice of $l$, it also holds that $S_{2} \backslash\left(S_{1} \cap S_{2}\right)$ is isomorphic to an $\mathbb{A}^{1}$-bundle over $C_{4} \backslash\left(S_{1} \cap C_{4}\right)$. Hence the condition (1) holds for the triplet $\left(S_{1}, S_{2}, C_{3}\right)$.

As a result, the triplet $\left(S_{1}, S_{2}, C_{d}\right)$ satisfies the conditions (I)-(III), (I') and (1) for $d=3,4$.

Proposition 4.7. There exists a triplet $\left(V, D_{1} \cup D_{2}, U\right)$ with $(\dagger)$ such that $V$ is a blow-up of $\mathbb{P}^{3}$ along a smooth rational curve of degree $d$ for $d=1,2,3,4$.

Proof. We define $S_{1}$ and $S_{2}$ in $\mathbb{P}_{[x: y: z: t]}^{3}$ as

$$
\begin{aligned}
& S_{1}:=\{x=0\} \\
& S_{2}:=\left\{x^{2} z+y^{3}+x y t=0\right\} .
\end{aligned}
$$

Since $S_{1}$ is a plane and $S_{2}$ is a non-normal irreducible cubic surface, they satisfy the conditions (II) and (I'). We write the conductor locus of $S_{2}$ as $E_{S_{2}}$. Then we have

$$
E_{S_{2}}=\{x=y=0\}=S_{1} \cap S_{2},
$$

which implies that the condition (III) holds for $S_{1}$ and $S_{2}$.

Let $\psi: X \rightarrow \mathbb{P}^{3}$ be the blow-up along $E_{S_{2}}$. Then it is easy to see that $\psi_{*}^{-1}\left(S_{2}\right)$ is smooth and hence $\left.\psi\right|_{S_{2}}$ is the normalization of $S_{2}$.

Since $S_{2}$ contains three lines $E_{S_{2}},\{y=z=0\}$ and $\{x=y, x+z+t=0\}$ which are not concurrent, $S_{2}$ is not a cone. Hence $S_{2}$ belongs to the class $(C)$ of [A-F83. In particular, $\psi_{*}^{-1}\left(S_{2}\right)$ is isomorphic to $\mathbb{F}_{1}$ and $\left.E_{\psi}\right|_{\psi_{*}^{-1}\left(S_{2}\right)} \sim$ $\Sigma_{1}+f_{1}$. We note that $\left.E_{\psi}\right|_{\psi_{*}^{-1}\left(S_{2}\right)}$ is reducible since $S_{2} \backslash E_{S_{2}} \cong \mathbb{A}^{2}$.

On $\psi_{*}^{-1}\left(S_{2}\right) \cong \mathbb{F}_{1}$, we take general smooth members

$$
\widetilde{C}_{1} \in\left|f_{1}\right|, \widetilde{C}_{2} \in\left|\Sigma_{1}+f_{1}\right|, \widetilde{C}_{3} \in\left|\Sigma_{1}+2 f_{1}\right| \text { and } \widetilde{C}_{4} \in\left|2 \Sigma_{1}+2 f_{1}\right|
$$

such that $\widetilde{C}_{d} \cap E_{\psi}$ consists of a single point for $d=1,2,3$, 4 . Since $\Sigma_{1}+2 f_{1}$ is very ample, we can choose $\widetilde{C}_{3}$ such that its tangent line of $\widetilde{C}_{3}$ at $q:=\widetilde{C}_{3} \cap E_{\psi}$ is different from the ruling of $E_{\psi}$ containing $q$.

Fix $d \in\{1,2,3,4\}$. By construction, the curve

$$
C_{d}:=\psi\left(\widetilde{C}_{d}\right)
$$


is smooth and rational of degree $d$. Since $\widetilde{C}_{d} \cap E_{\psi}$ consists of a single point, so does $C_{d} \cap E_{S_{2}}=C_{d} \cap S_{1}$. Hence the triplet $\left(S_{1}, S_{2}, C_{d}\right)$ satisfies the condition (I). As $C_{d} \backslash\left(S_{1} \cap C_{d}\right) \cong \mathbb{A}^{1}$ and $S_{2} \backslash\left(S_{1} \cap S_{2}\right) \cong \mathbb{A}^{2}$, the triplet satisfies the condition (1). Hence the triplet $\left(S_{1}, S_{2}, C_{d}\right)$ satisfies the conditions (I)-(III), (I') and (1).

4.3. The type (A3). We construct triplets $\left(V, D_{1} \cup D_{2}, U\right)$ with $(\dagger)$ of type (A3).

Proposition 4.8. There exists a triplet $\left(V, D_{1} \cup D_{2}, U\right)$ with $(\dagger)$ such that $V$ is a blow-up of $\mathbb{Q}^{3}$ along a smooth rational curve of degree $d$ for $d=1,3,4$.

Proof. First, we construct hypersurfaces $S_{1}$ and $S_{2}$ in $\mathbb{Q}^{3}$ which compose desired triplets as in Lemma 4.3. Let $l$ be a line in $\mathbb{Q}^{3}$ and $\psi_{1}: X \rightarrow \mathbb{Q}^{3}$ the blow-up of $\mathbb{Q}^{3}$ along $l$. Then $X$ is a Fano 3-fold of No.31 in M-M81, Table $2]$ and there is the extremal contraction $\psi_{2}: X \rightarrow \mathbb{P}^{2}$ different from $\psi_{1}$ and

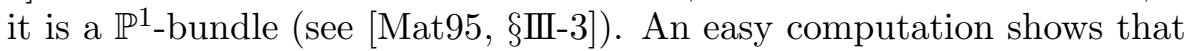

$$
\psi_{2}^{*} \mathcal{O}_{\mathbb{P}^{2}}(1) \sim \psi_{1}^{*} \mathcal{O}_{\mathbb{Q}^{3}}(1)-E_{\psi_{1}} .
$$

We also obtain that $N_{l} \mathbb{Q}^{3}=\mathcal{O}_{l} \oplus \mathcal{O}_{l}(1)$. Hence $E_{\psi_{1}} \cong \mathbb{F}_{1}$ and $\left.\psi_{2}\right|_{E_{\psi_{1}}}: E_{\psi_{1}} \rightarrow$ $\mathbb{P}^{2}$ is a blow-up at a point. Let $p$ be a center of blow-up $\left.\psi_{2}\right|_{E_{\psi_{1}}}$. Let $r_{1} \in$ $\left|\mathcal{O}_{\mathbb{P}^{2}}(1) \otimes \mathcal{I}_{p}\right|$ and $r_{2} \in\left|\mathcal{O}_{\mathbb{P}^{2}}(2) \otimes \mathcal{I}_{p}\right|$ be smooth curves such that $r_{1} \cap r_{2}=$ $\{p\}$. We take $\widetilde{S}_{i}$ and $S_{i}$ for $i=1,2$ as

$$
\widetilde{S}_{i}:=\psi_{2}^{-1}\left(r_{i}\right) \text { and } S_{i}:=\psi_{1}\left(\widetilde{S}_{i}\right) .
$$

Then the pair $\left(S_{1}, S_{2}\right)$ satisfies the condition (I').

Claim 1. It holds that $S_{1} \cong \mathbb{Q}_{0}^{2}$ and $S_{2}$ is a non-normal Gorenstein del Pezzo surface of class (C) in $\mathrm{A}-\mathrm{F} 83$.

Proof of Claim 1. By (4.3.1), we have $S_{i} \in\left|\mathcal{O}_{\mathbb{Q}^{3}}(i) \otimes \mathcal{I}_{l}^{i}\right|$ for $i=1$, 2. Hence it suffices to show that $\widetilde{S}_{1} \cong \mathbb{F}_{2}$ to obtain the first assertion. We also note that $S_{2}$ is a non-normal Gorenstein del Pezzo surface of degree 4. By Lemma 2.2 and $\mathrm{A}-\mathrm{F} 83$, $S_{2}$ belongs to

$$
\begin{cases}\text { the class }(\mathrm{C}) \text { of }[\mathrm{A}-\mathrm{F} 83] & \text { if } \widetilde{S}_{2} \cong \mathbb{F}_{2} \text { and } \\ \text { the class (D) of }[\mathrm{A}-\mathrm{F} 83] & \text { if } \widetilde{S}_{2} \cong \mathbb{F}_{0} .\end{cases}
$$

Hence it suffices to show that $\widetilde{S}_{2} \cong \mathbb{F}_{2}$ to obtain the second assertion. Note that $\psi_{1}\left(E_{\psi_{1}} \cap \widetilde{S}_{2}\right)$ is the conductor locus $E_{S_{2}}=l$ of $S_{2}$.

Fix $i \in\{1,2\}$. Since $N_{E_{\psi_{1}}} X=\mathcal{O}_{E_{\psi_{1}}}\left(-\Sigma_{1}\right)$ and $\left.\widetilde{S}_{i}\right|_{E_{\psi_{1}}} \sim i\left(\Sigma_{1}+f_{1}\right)$, we have

$$
\left(E_{\psi_{1}}^{2} \cdot \widetilde{S}_{i}\right)=-i\left(\Sigma_{1} \cdot \Sigma_{1}+f_{1}\right)_{E_{\psi_{1}}}=0 .
$$

By the choice of $r_{i}$, the intersection $\left.\widetilde{S}_{i}\right|_{E_{\psi_{1}}}$ is decomposed to two curves $\Sigma_{1}$ and $C \sim(i-1) \Sigma_{1}+i f_{1}$ on $E_{\psi_{1}}$. Note that $\Sigma_{1}$ and $C$ are a ruling and a section on $\widetilde{S}_{i}$ respectively. By (4.3.2), we have

$$
0=\left(\Sigma_{1}+C\right)^{2}=\Sigma_{1}^{2}+2\left(\Sigma_{1} \cdot C\right)+C^{2}=2+C^{2} \text { on } \widetilde{S}_{i} .
$$

Hence $C^{2}=-2$ and $S_{i} \cong \mathbb{F}_{2}$. 
Hence $\mathbb{Q}^{3} \backslash S_{1} \cong \mathbb{A}^{3}$ by [Fur93, Theorem A] and the condition (II) holds for $S_{1}$ and $S_{2}$. By the choice of $r_{1}$ and $r_{2}$, we have $S_{1} \cap S_{2}=\psi_{1}\left(\widetilde{S}_{1} \cap \widetilde{S}_{2}\right)=l$. Since

$$
\left(\left.\psi_{1}\right|_{\widetilde{S}_{2}}\right)^{-1}(l)=\left.E_{\psi_{1}}\right|_{\widetilde{S}_{2}}=\Sigma_{2}+C
$$

for some ruling $C$ of $\widetilde{S}_{2} \cong \mathbb{F}_{2}$, we have $S_{2} \backslash\left(S_{1} \cap S_{2}\right) \cong \mathbb{F}_{2} \backslash\left(\Sigma_{2} \cup C\right) \cong \mathbb{A}^{2}$. Hence the condition (III) holds for $S_{1}$ and $S_{2}$.

Next we construct desired centers of blow-ups. On $\widetilde{S}_{2} \cong \mathbb{F}_{2}$, we take general smooth members

$$
\widetilde{C}_{1} \in\left|f_{2}\right|, \widetilde{C}_{3} \in\left|\Sigma_{2}+2 f_{2}\right| \text { and } \widetilde{C}_{4} \in\left|\Sigma_{2}+3 f_{2}\right| .
$$

Since $\Sigma_{2}+3 f_{2}$ is very ample on $\mathbb{F}_{2}$, we can choose $\widetilde{C}_{4}$ such that $\widetilde{C}_{4}$ contains $q:=\Sigma_{2} \cap C$ and its tangent line at $q$ is different from that of the ruling of $E_{\psi_{1}} \cong \mathbb{F}_{1}$ containing $q$.

Fix $d \in\{1,3,4\}$. By construction, the curve

$$
C_{d}:=\psi_{1}\left(\widetilde{C}_{d}\right)
$$

is smooth and rational of degree $d$. Since $\widetilde{C}_{d} \cap E_{\psi_{1}}=\widetilde{C}_{d} \cap\left(\Sigma_{2} \cup C\right)$ consists of a single point, so does $C_{d} \cap l=C_{d} \cap S_{1}$. Hence the triplet $\left(S_{1}, S_{2}, C_{d}\right)$ satisfies the condition (I). As $C_{d} \backslash\left(S_{1} \cap C_{d}\right) \cong \mathbb{A}^{1}$ and $S_{2} \backslash\left(S_{1} \cap S_{2}\right) \cong \mathbb{A}^{2}$, the triplet also satisfies the condition (1). Hence the triplet $\left(S_{1}, S_{2}, C_{d}\right)$ satisfies the conditions (I)-(III), (I') and (1).

Proposition 4.9. There exists a triplet $\left(V, D_{1} \cup D_{2}, U\right)$ with $(\dagger)$ such that $V$ is a blow-up of $\mathbb{Q}^{3}$ along a conic.

Proof. We use the notation $l, \psi_{1}, \psi_{2}, p, r_{1}, \widetilde{S}_{1}$ and $S_{1}$ as in Proposition 4.8 . We take a smooth conic $r_{2} \subset \mathbb{P}^{2}$ such that $r_{1} \cap r_{2}$ consists of a single point which is different from $p$. We take

$$
\widetilde{S}_{2}:=\psi_{2}^{-1}\left(r_{2}\right) \text { and } S_{2}:=\psi_{1}\left(\widetilde{S}_{2}\right) .
$$

Then the pair $\left(S_{1}, S_{2}\right)$ satisfies the conditions (II) and (I').

A computation as in the proof of Claim 1 shows that $S_{2}$ belongs to the class (D) of A-F83. More precisely, we have $\widetilde{S}_{2} \cong \mathbb{F}_{0},\left.E_{\psi_{1}}\right|_{\widetilde{S}_{2}} \sim \Sigma_{0}$ and

$$
\left(\left.\psi_{1}\right|_{\widetilde{S}_{2}}\right)^{-1}\left(S_{1} \cap S_{2}\right)=\left.E_{\psi_{1}}\right|_{\widetilde{S}_{2}}+C
$$

for some ruling $C \in\left|f_{0}\right|$ on $\widetilde{S}_{2} \cong \mathbb{F}_{0}$. Hence $S_{2} \backslash\left(S_{1} \cap S_{2}\right) \cong \widetilde{S}_{2} \backslash\left(E_{\psi_{1}} \cup C\right) \cong$ $\mathbb{A}^{2}$ and the condition (III) holds for $S_{1}$ and $S_{2}$.

We take curves

$$
\widetilde{C}_{2} \in\left|\Sigma_{0}\right| \text { and } C_{2}:=\psi_{1}\left(\widetilde{C}_{2}\right)
$$

such that $\widetilde{C}_{2} \neq\left. E_{\psi_{1}}\right|_{\widetilde{S}_{2}}$. Then $C_{2}$ is a smooth conic. Since $\widetilde{C}_{2} \cap\left(\left.E_{\psi_{1}}\right|_{\widetilde{S}_{2}} \cup C\right)$ consists of a single point, so does $C_{2} \cap S_{1}$. Hence the triplet $\left(S_{1}, S_{2}, C_{2}\right)$ satisfies the conditions (I) and (1) as in the proof of Proposition 4.8 ,

4.4. The type (A4). We construct triplets $\left(V, D_{1} \cup D_{2}, U\right)$ with $(\dagger)$ of type (A4).

Proposition 4.10. There exists a triplet $\left(V, D_{1} \cup D_{2}, U\right)$ with $(\dagger)$ such that $V$ is a blow-up of $V_{5}$ along a line. 
Proof. We take hyperplane sections

$$
S_{1}=H_{5}^{0} \text { and } S_{2}=H_{5}^{\infty}
$$

as in [Fur00. Then the pair $\left(S_{1}, S_{2}\right)$ satisfies the condition (I'). Note that $S_{2}$ is non-normal. By [Fur00, Lemma 7], the normalization $\bar{S}_{2}$ of $S_{2}$ is isomorphic to $\mathbb{F}_{3}$. Hence $S_{2}$ belongs to the class (C) of A-F83 and the conductor locus $\bar{E}_{S_{2}}$ of $\bar{S}_{2}$ is decomposed as $\Sigma_{3} \cup f_{3}$. Since the intersection $S_{1} \cap S_{2}$ is the conductor locus of $S_{2}$ by [Fur00, Lemma 11], we have $S_{2} \backslash$ $\left(S_{1} \cap S_{2}\right) \cong \mathbb{F}_{3} \backslash\left(\Sigma_{3} \cup f_{3}\right) \cong \mathbb{A}^{2}$. Hence the condition (III) holds for $S_{1}$ and $S_{2}$. The pair also satisfies the condition (II) since $V_{5} \backslash S_{1} \cong \mathbb{A}^{3}$ by Fur00, Lemma12].

We take $\widetilde{C}_{1} \subset \bar{S}_{2}$ and $C_{1} \subset S_{2}$ as

$$
\widetilde{C}_{1} \in\left|f_{3}\right| \text { with } \widetilde{C}_{1} \not \subset \bar{E}_{S_{2}} \text { and } C_{1}:=\sigma_{S_{2}}\left(\widetilde{C}_{1}\right),
$$

where $\sigma_{S_{2}}: \bar{S}_{2} \rightarrow S_{2}$ is the normalization. Then $C_{1}$ is a line in $S_{2}$ different from $E_{S_{2}}$ and hence the triplet $\left(S_{1}, S_{2}, C_{1}\right)$ satisfies the condition (I). As $C_{1} \backslash\left(S_{1} \cap C_{1}\right) \cong \mathbb{A}^{1}$ and $S_{2} \backslash\left(S_{1} \cap S_{2}\right) \cong \mathbb{A}^{2}$, the triplet $\left(S_{1}, S_{2}, C_{1}\right)$ also satisfies the condition (1). Hence the triplet $\left(S_{1}, S_{2}, C_{1}\right)$ satisfies the conditions (I)-(I II), (I') and (1).

Proposition 4.11. There exists a triplet $\left(V, D_{1} \cup D_{2}, U\right)$ with $(\dagger)$ such that $V$ is a blow-up of $V_{5}$ along a smooth rational curve of degree $d$ for $d=2,3$.

Proof. We take hyperplane sections

$$
S_{1}=H_{5}^{\infty} \text { and } S_{2}=H_{5}^{0}
$$

as in [Fur00. Then the pair $\left(S_{1}, S_{2}\right)$ satisfies the condition (I'). By Fur00, Lemma 12], we have $V_{5} \backslash S_{1} \cong \mathbb{A}^{3}$ and $S_{2} \backslash\left(S_{1} \cap S_{2}\right) \cong \mathbb{A}^{2}$. Hence $S_{1}$ and $S_{2}$ satisfy the conditions (II) and (III).

Fix $d \in\{2,3\}$ and take $C_{d}$ as a smooth rational curve of degree $d$ in $S_{2}$ as in [Kis05, Lemma 2.3]. Since $S_{1} \cap S_{2}$ is a line, the triplet $\left(S_{1}, S_{2}, C_{d}\right)$ satisfies the condition (I). As $C_{d} \backslash\left(S_{1} \cap C_{d}\right) \cong \mathbb{A}^{1}$ and $S_{2} \backslash\left(S_{1} \cap S_{2}\right) \cong \mathbb{A}^{2}$ by construction, the triplet $\left(S_{1}, S_{2}, C_{d}\right)$ satisfies the condition (1).

4.5. Another example of type (A3). We give an example of triplets with $(\dagger)$ of type (A3) such that the image of its boundary divisors by the blow-up morphism does not satisfy the condition (II) of Lemma 4.3.

Example 4.12. Take $S_{1}, S_{2}$ and $C$ in $\mathbb{Q}^{3}=\left\{X_{1}^{2}+X_{0} X_{4}+X_{2} X_{3}=0\right\} \subset$ $\mathbb{P}_{\left[X_{0}: \cdots: X_{4}\right]}^{4}$ as

$$
\begin{aligned}
S_{1} & :=\left\{X_{1}^{2}+X_{0} X_{4}+X_{2} X_{3}=0=X_{1}=0\right\} \cong \mathbb{P}^{1} \times \mathbb{P}^{1}, \\
S_{2} & :=\left\{X_{1}^{2}+X_{0} X_{4}+X_{2} X_{3}=0=X_{0}^{2}+X_{1} X_{2}=0\right\}, \text { and } \\
C & :=\left\{X_{0}=X_{1}=-X_{2}=X_{3}-X_{4}\right\} .
\end{aligned}
$$

Then $S_{1} \cong \mathbb{P}^{1} \times \mathbb{P}^{1}$. Since $\mathbb{Q}^{3} \backslash S_{1} \cong \mathrm{SL}(2 ; \mathbb{C})$, the triplet $\left(S_{1}, S_{2}, C\right)$ does not satisfy the condition (II).

Let $g: V \rightarrow \mathbb{Q}^{3}$ be the blow-up of $\mathbb{Q}^{3}$ along the line $C$ and $D_{i}:=g_{*}^{-1}\left(S_{i}\right)$ for $i=1,2$. Then the open set $U:=V \backslash\left(D_{1} \cup D_{2}\right)$ is the affine modification 
of $\left\{1+x_{0} x_{4}+x_{2} x_{3}=0\right\}$ with the locus

$$
\left(\begin{array}{l}
\left(\left\{1+x_{0} x_{4}+x_{2} x_{3}=0, x_{0}^{2}+x_{2}=0, x_{0}=1\right\}\right. \\
\subset\left\{1+x_{0} x_{4}+x_{2} x_{3}=0, x_{0}^{2}+x_{2}=0\right\}
\end{array}\right)
$$

in $\mathbb{A}_{\left(x_{0}, x_{2}, x_{3}, x_{4}\right)}^{4}$, which is isomorphic to

$$
\begin{aligned}
& \left\{1+x_{0} x_{4}+x_{2} x_{3}=0,\left(x_{0}^{2}+x_{2}\right) w=x_{0}-1\right\} \\
\cong & \left\{1+x_{0} x_{4}+\left(x_{2}-x_{0}^{2}\right) x_{3}=0, x_{0}=1+x_{2} w\right\} \\
\cong & \left\{1+x_{0}\left(x_{4}-x_{0} x_{3}\right)+x_{2} x_{3}=0, x_{0}=1+x_{2} w\right\} \\
\cong & \left\{1+x_{0} x_{4}+x_{2} x_{3}=0, x_{0}=1+x_{2} w\right\} \\
\cong & \left\{1+x_{4}+x_{2}\left(x_{3}+x_{4} w\right)=0, x_{0}=0\right\} \\
\cong & \left\{x_{4}=x_{0}=0\right\} \cong \mathbb{A}^{3}
\end{aligned}
$$

in $\mathbb{A}_{\left(x_{0}, x_{2}, x_{3}, x_{4}, w\right)}^{5}$. As $S_{1}$ and $S_{2}$ satisfy the conditions (I) and (I'), we have $K_{V}+D_{1}+D_{2}=0$. Hence $\left(V, D_{1} \cup D_{2}, U\right)$ is a triplet with ( $\dagger$ ) of type (A3).

\section{Construction of examples of type (B)}

In this section, we construct examples of triplets with $(\dagger)$ of type (B1)(B3).

5.1. The characterization of $\mathbb{A}^{3}$. We review the characterization of $\mathbb{A}^{3}$ by Sh. Kaliman.

Theorem 5.1 (cf. Kal02]). Let $X$ be an affine 3-fold such that

(0) $\operatorname{Pic} X=0$ and all invertible functions on $X$ are constants;

(1) The Euler characteristic of $X$ is $\chi_{\mathrm{top}}(X)=1$;

(2) There exists a Zariski open subset $Z$ of $X$ and a morphism $p: Z \rightarrow r$ onto a curve $r$ whose fibers are isomorphic to $\mathbb{A}^{2}$;

(3) Each irreducible component of $X \backslash Z$ has the trivial Picard group.

Then $X$ is isomorphic to $\mathbb{A}^{3}$.

5.2. The type (B3). With Theorem 5.1, we can construct a triplet $\left(V, D_{1} \cup\right.$ $\left.D_{2}, U\right)$ with ( $\dagger$ ) of type (B3).

Proposition 5.2. Let $x_{1}, x_{2}, x_{3}$ and $y$ be coordinates of $\mathbb{P}:=\mathbb{P}(1,1,1,2)$ of degree 1,1,1 and 2 respectively. Define $S_{1}$ and $S_{2} \subset \mathbb{P}$ as

$$
\begin{aligned}
& S_{1}:=\{y=0\} \text { and } \\
& S_{2}:=\left\{y x_{1}+x_{2} x_{3}\left(x_{2}+x_{3}\right)=0\right\} .
\end{aligned}
$$

Let $\varphi: V \rightarrow \mathbb{P}:=\mathbb{P}(1,1,1,2)$ be the blow-up of $\mathbb{P}$ at the vertex and $D_{i}:=$ $\varphi_{*}^{-1}\left(S_{i}\right)$ for $i=1,2$. Then the following holds:

(1) $K_{V}+D_{1}+D_{2}=0$.

(2) $D_{1}$ and $D_{2}$ are $\mathbb{Z}$-basis of $\operatorname{Pic} V$.

(3) $E_{\varphi} \backslash\left(D_{2} \cap E_{\varphi}\right) \cong \mathbb{A}^{2}$.

(4) $\mathbb{P} \backslash\left(S_{1} \cup S_{2}\right) \cong \mathbb{A}^{2} \times \mathbb{C}^{*}$.

(5) $V \backslash\left(D_{1} \cup D_{2}\right) \cong \mathbb{A}^{3}$.

In particular, $\left(V, D_{1} \cup D_{2}, \mathbb{A}^{3}\right)$ is a triplet with ( $\dagger$ ) of type (B3). 
Proof. (1): Let $H:=\varphi^{*} \mathcal{O}_{\mathbb{P}}(2)$, then $D_{1} \sim H$. By the Jacobian criterion, the vertex $p$ of $V$ is the unique singular point of $S_{2}$ and $\left(S_{2}, p\right)$ is an $A_{1}$ singularity. Hence $\left.E_{\varphi}\right|_{D_{2}}$ is a (-2)-curve. We write $D_{2} \sim a H+b E_{\varphi}$. Then we have $4 a=H^{2} \cdot D_{2}=6$ and $4 b=E_{\varphi}^{2} \cdot D_{2}=-2$. Hence we have $D_{2} \sim \frac{3}{2} H-\frac{1}{2} E_{\varphi}$, which proves (1).

(2): Let $F$ be the pullback of $\mathcal{O}_{\mathbb{P}^{2}}(1)$ by the $\mathbb{P}^{1}$-bundle structure of $V=$ $\mathbb{P}_{\mathbb{P}^{2}}(\mathcal{O} \oplus \mathcal{O}(2))$. By Theorem 3.1 and (1), we have $D_{2} \sim H+F$ and Pic $V=$ $\mathbb{Z}[H] \oplus \mathbb{Z}[F]=\mathbb{Z}\left[D_{1}\right] \oplus \mathbb{Z}\left[D_{2}\right]$.

(3): Since $\left(E_{\varphi} \cdot D_{2}^{2}\right)=1$, the intersection $\left.D_{2}\right|_{E_{\varphi}}$ is a line in $E_{\varphi} \cong \mathbb{P}^{2}$ and hence $E_{\varphi} \backslash\left(D_{2} \cap E_{\varphi}\right) \cong \mathbb{A}^{2}$.

(4): Let $\alpha$ be the involution on $\mathbb{A}_{\left(x_{1}, x_{2}, x_{3}\right)}^{3}$ which sends $a$ to $-a$. Let $\pi: \mathbb{A}^{3} \rightarrow \mathbb{A}^{3} /\{\mathrm{id}, \alpha\}$ be the quotient morphism. By regarding $\{y \neq 0\} \subset \mathbb{P}$ as $\mathbb{A}^{3} /\{$ id, $\alpha\}$, we have an isomorphism

$$
\mathbb{P} \backslash\left(S_{1} \cup S_{2}\right) \cong\left(\mathbb{A}^{3} /\{\mathrm{id}, \alpha\}\right) \backslash \pi\left(\left\{x_{1}+x_{2} x_{3}\left(x_{2}+x_{3}\right)=0\right\}\right) .
$$

Consider the polynomial automorphism $\beta$ of $\mathbb{A}^{3}$ such that

$$
\beta\left(x_{1}\right)=x_{1}-x_{2} x_{3}\left(x_{2}+x_{3}\right), \beta\left(x_{2}\right)=x_{2}, \beta\left(x_{3}\right)=x_{3} .
$$

Since $\alpha$ commutes with $\beta$, we have the desired isomorphism

$$
\begin{aligned}
\mathbb{P} \backslash\left(S_{1} \cup S_{2}\right) & \cong\left(\mathbb{A}^{3} /\{\mathrm{id}, \alpha\}\right) \backslash \pi\left(\left\{\beta\left(x_{1}+x_{2} x_{3}\left(x_{2}+x_{3}\right)\right)=0\right\}\right) \\
& \cong\left(\mathbb{A}^{3} /\{\mathrm{id}, \alpha\}\right) \backslash \pi\left(\left\{x_{1}=0\right\}\right) \\
& \cong\left(\mathbb{A}_{\left(x_{2}, x_{3}\right)}^{2} \times \mathbb{C}_{\left(x_{1}\right)}^{*}\right) /\{\mathrm{id}, \alpha\} \\
& \cong \mathbb{A}^{2} \times \mathbb{C}^{*}
\end{aligned}
$$

(5): By (1), the compliment $V \backslash\left(D_{1} \cup D_{2}\right)$ is affine. By (2) and Fuj82, Proposition 1.1(1)], the condition (0) of Theorem 5.1 holds for $V \backslash\left(D_{1} \cup D_{2}\right)$. By (3) and (4), we have $\chi_{\text {top }}\left(V \backslash\left(D_{1} \cup D_{2}\right)\right)=\chi_{\text {top }}\left(E_{\varphi} \backslash\left(D_{2} \cap E_{\varphi}\right)\right)+$ $\chi_{\text {top }}\left(\mathbb{P} \backslash\left(S_{1} \cup S_{2}\right)\right)=\chi_{\text {top }}\left(\mathbb{A}^{2}\right)+\chi_{\text {top }}\left(\mathbb{A}^{2} \times \mathbb{C}^{*}\right)=1$. Hence the condition (1) of Theorem 5.1 holds for $V \backslash\left(D_{1} \cup D_{2}\right)$. Applying Theorem 5.1 by setting $X:=V \backslash\left(D_{1} \cup D_{2}\right)$ and $Z:=V \backslash\left(D_{1} \cup D_{2} \cup E_{\varphi}\right) \cong \mathbb{P} \backslash\left(S_{1} \cup S_{2}\right)$, we obtain (5).

5.3. The types (B1) and (B2). To construct examples of triplets with $(\dagger)$ of type (B1) and (B2), we use the following lemma, which is a corollary of Theorem 5.1 .

Lemma 5.3. Let $\varphi: V \rightarrow \mathbb{P}^{2}$ be a $\mathbb{P}^{1}$-bundle. Let $D_{1}$ and $D_{2} \subset V$ be irreducible and generically birational sections of $\varphi$ which satisfy the following:

(0') $D_{1}$ and $D_{2}$ are $\mathbb{Z}$-basis of $\operatorname{Pic} V$ and $D_{1}+D_{2}$ is ample.

(1') There is the unique point $p \in \mathbb{P}^{2}$ such that $\varphi^{-1}(p) \subset D_{1} \cup D_{2}$.

(2') $\varphi\left(D_{1} \cap D_{2}\right) \subset \mathbb{P}^{2}$ is a line containing $p$.

Then the open subvariety $U:=V \backslash\left(D_{1} \cup D_{2}\right)$ is isomorphic to $\mathbb{A}^{3}$.

Proof. Since $D_{1}+D_{2}$ is ample, the variety $U$ is affine. By Fuj82, Proposition 1.18 (1)], the condition (0') implies the condition (0) of Theorem 5.1 for $U$.

Let $l:=\varphi\left(D_{1} \cap D_{2}\right)$. Then $V_{0}:=V \backslash\left(\varphi^{-1}(l) \cup D_{1}\right)$ is $\mathbb{A}^{1}$-bundle over $\mathbb{A}^{2}$ by the condition (1') and Miy78, §4.1]. Since $V_{0} \cap D_{2}$ is a section of $V_{0}$ by 
$\left(1^{\prime}\right)$ and $\left(2^{\prime}\right)$, it is easy to check that

$$
U \cap\left(V \backslash \varphi^{-1}(l)\right) \cong V_{0} \cap\left(V \backslash D_{2}\right) \cong \mathbb{A}^{2} \times \mathbb{C}^{*} .
$$

Note that the condition (2') implies that $U \cap \varphi^{-1}(l) \cong \mathbb{A}^{2}$. Hence $U$ satisfies the conditions (2) and (3) of Theorem 5.1 if we set $Z:=U \cap\left(V \backslash \varphi^{-1}(l)\right)$.

Since $\chi_{\text {top }}(U)=\chi_{\text {top }}(Z)+\chi_{\text {top }}(U \backslash Z)=1$, the condition (1) of Theorem 5.1 is also satisfied. Hence we have the assertion by Theorem 5.1 .

Remark 5.4. The conditions as in Lemma 5.3 is not necessary to construct triplets $\left(V, D_{1} \cup D_{2}, U\right)$ with (†) of type (B). For example, let $\left(V, D_{1} \cup D_{2}, U\right)$ be a triplet as in Proposition 5.2. The intersection $D_{1} \cap D_{2}$ consists of three curves, but the conditions (1') and (2') implies the intersection consists of at most two curves.

We can now construct examples of triplets with ( $\dagger$ ) of type (B1) and (B2).

Proposition 5.5. There exist divisors $D_{1}$ and $D_{2}$ in a smooth 3-fold $V \subset$ $\mathbb{P}^{2} \times \mathbb{P}^{2}$, which is a divisor of bidegree $(1,2)$, such that $\left(V, D_{1} \cup D_{2}, \mathbb{A}^{3}\right)$ is a triplet with $(\dagger)$ of type (B1).

Proof. In $\mathbb{P}_{\left[x_{0}: x_{1}: x_{2}\right]}^{2} \times \mathbb{P}_{\left[y_{0}: y_{1}: y_{2}\right]}^{2}$, take $V, D_{1}$ and $D_{2}$ as

$$
\begin{aligned}
V & =\left\{x_{0} y_{0}^{2}+x_{1} y_{1}^{2}+x_{2} q\left(y_{0}, y_{1}, y_{2}\right)=0\right\} \\
D_{1} & :=\left\{x_{0} y_{0}^{2}+x_{1} y_{1}^{2}+x_{2} q\left(y_{0}, y_{1}, y_{2}\right)=x_{0} y_{1}+x_{2}\left(b y_{0}+a y_{1}\right)=0\right\} \\
D_{2} & :=\left\{x_{0} y_{0}^{2}+x_{1} y_{1}^{2}+x_{2} q\left(y_{0}, y_{1}, y_{2}\right)=x_{2}=0\right\}
\end{aligned}
$$

with $a \in \mathbb{C}$ and $b \in \mathbb{C}^{*}$ and where $q$ is a quadric form with $q([0: 0: 1]) \neq 0$. It is easy to check the smoothness of $V$ by the Jacobian criterion. Let $\varphi:=\operatorname{pr}_{2}: V \rightarrow \mathbb{P}^{2}$, which is a $\mathbb{P}^{1}$-bundle. We note that $\operatorname{Pic} V$ is generated by $H_{1}:=\operatorname{pr}_{1}^{*} \mathcal{O}_{\mathbb{P}^{2}}(1)$ and $H_{2}:=\operatorname{pr}_{2}^{*} \mathcal{O}_{\mathbb{P}^{2}}(1)$ by the Grothendieck-Lefschetz theorem. Since $D_{1} \sim H_{1}+H_{2}$ and $D_{2} \sim H_{1}, D_{1}$ and $D_{2}$ satisfy the condition $\left(0^{\prime}\right)$ of Lemma 5.3 and $(\dagger)$. An easy computation shows that $D_{1} \cap D_{2}=\left\{x_{0}=\right.$ $\left.y_{0}=y_{1}=0\right\} \cup\left\{x_{0}=x_{2}=y_{1}=0\right\}$ and the condition (2') holds for $D_{1}$ and $D_{2}$.

For homogeneous elements $g_{0}, g_{1}$ and $g_{2} \in \mathbb{C}\left[y_{0}, y_{1}, y_{2}\right]$, a divisor $\{f=$ $\left.x_{0} g_{0}+x_{1} g_{1}+x_{2} g_{2}=0\right\}$ in $V$ contains $\varphi^{-1}([a: b: c])$ only if the rank of

$$
\left(\begin{array}{ccc}
a^{2} & b^{2} & q(a, b, c) \\
g_{0}(a, b, c) & g_{1}(a, b, c) & g_{2}(a, b, c)
\end{array}\right)
$$

is smaller than two. Hence we can check that $D_{1}$ and $D_{2}$ satisfy the condition (1') with $p:=[0: 0: 1]$.

Proposition 5.6. There exist divisors $D_{1}$ and $D_{2}$ in $\mathbb{P}^{1} \times \mathbb{P}^{2}$ such that $\left(\mathbb{P}^{1} \times \mathbb{P}^{2}, D_{1} \cup D_{2}, \mathbb{A}^{3}\right)$ is a triplet with ( $\dagger$ ) of type (B2).

Proof. In $V=\mathbb{P}_{\left[x_{0}: x_{1}\right]}^{1} \times \mathbb{P}_{\left[y_{0}: y_{1}: y_{2}\right]}^{2}$, we take $D_{1}$ and $D_{2}$ as

$$
\begin{aligned}
& D_{1}:=\left\{x_{0} y_{1}+x_{1} y_{2}=0\right\} \\
& D_{2}:=\left\{x_{0} y_{1}\left(a y_{1}+y_{2}\right)+x_{1}\left(b y_{1}^{2}+a y_{1} y_{2}+y_{2}^{2}\right)=0\right\}
\end{aligned}
$$

with $a \in \mathbb{C}$ and $b \in \mathbb{C}^{*}$. Since $D_{i}$ is of bidegree $(1, i)$ for $i=1,2$, the condition $\left(0^{\prime}\right)$ and $(\dagger)$ holds for $D_{1}$ and $D_{2}$. They also satisfy the condition (1') with $\varphi:=\operatorname{pr}_{2}$ and $p:=[1: 0: 0]$. This implies that $D_{1}$ and $D_{2}$ are irreducible. 
An easy computations shows that $D_{1} \cap D_{2}=\left\{y_{1}=y_{2}=0\right\} \cup\left\{x_{1}=y_{1}=0\right\}$. Hence $D_{1}$ and $D_{2}$ satisfy the condition (2') and we complete the proof.

Summarizing Propositions 4.6 4.11, 5.2, 5.5 and 5.6, we have proved Theorem $1.1(2)$.

In the remaining part of this article, we fix a triplet $\left(V, D_{1} \cup D_{2}, U\right)$ with $(\dagger)$. We shall prove Theorem 1.1 (1) to seek a contradiction to Lemma 3.5 when $V$ is of type neither (A) or (B). To obtain a contradiction, we compute $\chi_{\text {top }}\left(D_{1}\right), \chi_{\text {top }}\left(D_{2}\right)$ and $\chi_{\text {top }}\left(D_{1} \cap D_{2}\right)$. From now on, we use Notation 2 ,

\section{ExClusion OF IMPRIMITIVE FANO 3-FOLDS}

The aim of this section is to prove Theorem 1.1(1) when $V$ is imprimitive. For this reason, we assume that $V$ is the blow-up of a Fano variety $W$ of index $r$ along a smooth curve $C$. We use Notation 2 and fix $\varphi_{1}=\mathrm{Bl}_{C}$. By Proposition 3.4, we may assume that

$$
D_{1} \sim \varphi^{*} \mathcal{O}_{W}(1) \text { and } D_{2} \sim \varphi^{*} \mathcal{O}_{W}(r-1)-E_{\varphi} .
$$

Notation 4. We also use the following notation.

- $S_{i}:=\varphi_{1 *}\left(D_{i}\right)$ for $i=1,2$.

- $\tau: \widetilde{S}_{2} \rightarrow S_{2}$ : the composition of the normalization and the minimal resolution.

- $F:=\left(S_{1} \cap S_{2}\right)_{\text {red }}$.

- $J_{1}:=\left\{l:\right.$ a curve $\subset D_{1} \cap E_{\varphi_{1}} \mid \varphi_{1}(l)$ is a point $\}, N_{1}:=\sharp J_{1}$.

- $J_{2}:=\left\{l:\right.$ a curve $\subset D_{2} \cap E_{\varphi_{1}} \mid \varphi_{1}(l)$ is a point $\}, N_{2}:=\sharp J_{2}$.

- $J_{1 \cap 2}:=\left\{l:\right.$ a curve $\subset\left(D_{1} \cap D_{2}\right) \cap E_{\varphi_{1}} \mid \varphi_{1}(l)$ is a point $\}, N_{1 \cap 2}:=$ $\sharp J_{1 \cap 2}$.

By (6.0.1), we have $N_{1}=\sharp\left(C \cap S_{1}\right), N_{2}=\sharp\left(C \cap \operatorname{Sing} S_{2}\right)$ and $N_{1 \cap 2}=\sharp(C \cap$ $S_{1} \cap$ Sing $S_{2}$ ). We use Notation 1 when $S_{2}$ is non-normal.

6.1. The image of contraction $\varphi_{1}: V \rightarrow W_{1}$.

Lemma 6.1. It holds that $\chi_{\mathrm{top}}\left(D_{i}\right)=\chi_{\mathrm{top}}\left(S_{i}\right)+N_{i}$ for $i=1,2$, and $\chi_{\text {top }}\left(D_{1} \cap D_{2}\right)=\chi_{\text {top }}(F)+N_{1 \cap 2}$.

Proof. We have the first equations as follows:

$$
\begin{aligned}
\chi_{\mathrm{top}}\left(D_{i}\right) & =\chi_{\mathrm{top}}\left(D_{i} \backslash \bigcup_{l \in J_{i}} l\right)+\chi_{\mathrm{top}}\left(\bigcup_{l \in J_{i}} l\right) \\
& =\chi_{\mathrm{top}}\left(S_{i} \backslash \bigcup_{l \in J_{i}} \varphi(l)\right)+N_{i} \times \chi_{\mathrm{top}}\left(\mathbb{P}^{1}\right) \\
& =\chi_{\mathrm{top}}\left(S_{i}\right)+N_{i} .
\end{aligned}
$$

The second assertion follows from the same argument.

Proposition 6.2. We have the following:

(1) $\chi_{\text {top }}(F)=\chi_{\text {top }}\left(S_{1}\right)+\chi_{\text {top }}\left(S_{2}\right)+B_{3}\left(W_{1}\right)+2 p_{a}(C)+N_{1}+N_{2}-N_{1 \cap 2}-5$.

(2) $N_{1}+N_{2}-N_{1 \cap 2} \geq 1$ and the equality holds if and only if $\left(N_{1}, N_{2}, N_{1 \cap 2}\right)$ $=(1,0,0)$ or $(1,1,1)$. 
Proof. (1) The assertion holds by Lemma 3.5, Lemma 6.1 and the equation $B_{3}(V)=B_{3}\left(W_{1}\right)+2 p_{a}(C)$.

(2) The desired inequality is given by following inequalities:

$$
N_{1} \geq 1 \text { and } \max \left\{N_{1}, N_{2}\right\} \geq N_{1 \cap 2} .
$$

Lemma 6.3. It holds that $\chi_{\mathrm{top}}(F) \leq 1+\left(S_{1}^{2} \cdot S_{2}\right)$.

Proof. Since $\left.S_{1}\right|_{S_{2}} \subset S_{2}$ is ample, its support is connected. Since $S_{1} \subset W_{1}$ is ample, we have $B_{2}(F) \leq\left(S_{1}^{2} \cdot S_{2}\right)$. Hence $\chi_{\text {top }}(F) \leq B_{0}(F)+B_{2}(F) \leq$ $1+\left(S_{1}^{2} \cdot S_{2}\right)$.

Proposition 6.4. It holds that $W_{1}=\mathbb{P}^{3}, \mathbb{Q}^{3}$ or $V_{5}$.

Proof. By [M-M83, Proposition 5.12], it suffices to show that $W_{1}$ is not del Pezzo 3-fold $V_{d}$ of degree $1 \leq d \leq 4$. Suppose that $W_{1}=V_{d}$ for some $1 \leq d \leq 4$.

By (6.0.1), the surfaces $S_{1}$ and $S_{2}$ are hypersurfaces of $V_{d}$. By Proposition 6.2 and Lemma 6.3, we have

$$
1+d=1+\left(S_{1}^{2} \cdot S_{2}\right) \geq \chi_{\mathrm{top}}\left(S_{1}\right)+\chi_{\mathrm{top}}\left(S_{2}\right)+B_{3}\left(V_{d}\right)-4 .
$$

Together with Theorem 2.1, we have $3+d \geq B_{3}\left(V_{d}\right)$. Since

$$
B_{3}\left(V_{d}\right)= \begin{cases}42 & \text { if } d=1 \\ 20 & \text { if } d=2 \\ 10 & \text { if } d=3 \\ 4 & \text { if } d=4\end{cases}
$$

by [Isk80, Table 3.5], we have $d=4$. By Theorem 2.1 (4) and Lemma 2.2, we have $\chi_{\text {top }}\left(S_{i}\right) \geq 3$ for $i=1,2$. Hence (6.1.1) is rewritten as $5=1+d \geq 6$, a contradiction.

6.2. On the conditions in Lemma 4.3. Now we can prove the relations stated in Remark 4.5. Note that we use only Proposition 6.7 among propositions in this subsection to prove Theorem 1.1(1).

Proposition 6.5. The condition (II) in Lemma 4.3 holds for $\left(S_{1}, S_{2}, C\right)$ if and only if $B_{2}\left(S_{1}\right)$ is smallest possible among hyperplane sections of $W_{1}$.

Proof. By (6.0.1) and Proposition 6.4 the surface $S_{1}$ is a hyperplane section of $W_{1} \cong \mathbb{P}^{3}, \mathbb{Q}^{3}$ or $V_{5}$. By the classification of compactifications of contractible affine 3 -folds into Fano 3-folds with $B_{2}=1$ (see Kis05, Corollary 2.1]), we have the assertion.

Proposition 6.6. Suppose that the condition (II) in Lemma 4.3 holds for $\left(S_{1}, S_{2}\right)$. We also assume that $S_{2}$ is normal and rational. Then $S_{2} \backslash F$ is smooth.

Proof. By Proposition 6.4, we have $W_{1} \cong \mathbb{P}^{3}, \mathbb{Q}^{3}$ or $V_{5}$. Hence $S_{2}$ is a Gorenstein del Pezzo surface with $K_{S_{2}}^{2} \geq 3$ by (6.0.1). Let $S:=S_{2} \backslash F$ and Sing $S:=\left\{p_{1}, \ldots, p_{n}\right\}(n \geq 0)$. For all $i$, take an open ball $U_{i}^{\prime}:=\{x \in$ $\left.\mathbb{C}^{3} \mid d\left(x, p_{i}\right)<\epsilon\right\}$ with a sufficiently small $\epsilon>0$ such that $U_{i}^{\prime} \cap U_{j}^{\prime}=\emptyset$ for 
any $i \neq j$. Let $U_{i}:=U_{i}^{\prime} \cap S$. By the Mayer-Vietoris exact sequence, we have the following exact sequence of homologies:

$$
\begin{aligned}
\cdots & \rightarrow H_{2}(S \backslash \operatorname{Sing} S, \mathbb{Z}) \oplus \bigoplus_{i=1}^{n} H_{2}\left(U_{i}, \mathbb{Z}\right) \rightarrow H_{2}(S, \mathbb{Z}) \\
\rightarrow \bigoplus_{i=1}^{n} H_{1}\left(U_{i} \backslash\left\{p_{i}\right\}, \mathbb{Z}\right) & \rightarrow H_{1}(S \backslash \operatorname{Sing} S, \mathbb{Z}) \oplus \bigoplus_{i=1}^{n} H_{1}\left(U_{i}, \mathbb{Z}\right) \rightarrow \cdots .
\end{aligned}
$$

Since the singularity $\left(S, p_{i}\right)$ is a rational double point by [H-W81, Proposition 1.2], $U_{i}$ is contractible for any $i$ by Theorem 2.9. Applying Lemma 2.4 by setting $X=W \backslash S_{1}, S$ as above and $r=C \backslash\left(C \cap S_{1}\right)$, we have $H_{1}(S \backslash \operatorname{Sing} S, \mathbb{Z})=H_{1}(r \backslash(r \cap \operatorname{Sing} S), \mathbb{Z})$ and $H_{2}(S \backslash \operatorname{Sing} S, \mathbb{Z})=0$. Hence the exact sequence is rewritten as

$$
\cdots \rightarrow 0 \rightarrow H_{2}(S, \mathbb{Z}) \rightarrow \oplus_{i=1}^{n} H_{1}\left(U_{i} \backslash\left\{p_{i}\right\}, \mathbb{Z}\right) \rightarrow H_{1}(r \backslash(r \cap \operatorname{Sing} S), \mathbb{Z}) \rightarrow \cdots
$$

Since $S$ and $r \backslash(r \cap \operatorname{Sing} S)$ are affine, both $H_{2}(S, \mathbb{Z})$ and $H_{1}(r \backslash(r \cap \operatorname{Sing} S), \mathbb{Z})$ are free $\mathbb{Z}$-modules by [Ham83, Korollar]. Therefore $H_{1}\left(U_{i} \backslash\left\{p_{i}\right\}, \mathbb{Z}\right)$ is also a free module for any $i$.

Fix $i \in\{1, \ldots, n\}$. By the Hurewicz theorem, $H_{1}\left(U_{i} \backslash\left\{p_{i}\right\}, \mathbb{Z}\right)$ is the abelianization of the local fundamental group $\pi_{S, p_{i}}$ of $\left(S, p_{i}\right)$. By Theorem 2.7. $\pi_{S, p_{i}}$ is finite and so is $H_{1}\left(U_{i} \backslash\left\{p_{i}\right\}, \mathbb{Z}\right)$. Hence $H_{1}\left(U_{i} \backslash\left\{p_{i}\right\}, \mathbb{Z}\right)=0$ and $\pi_{S, p_{i}}$ is perfect. Hence $\left(S, p_{i}\right)$ is the $E_{8}$ singularity by Theorem 2.8. By Theorem 2.1 (1), it follows that

$$
\begin{aligned}
9 & \geq\left(9-K_{S_{2}}^{2}\right)+\chi_{\mathrm{top}}\left(\mathbb{P}^{2}\right)=\chi_{\mathrm{top}}\left(\widetilde{S}_{2}\right) \\
& \geq \chi_{\mathrm{top}}\left(S_{2}\right)+8 n \\
& \geq 3+8 n .
\end{aligned}
$$

Hence $n=0$ and we have the assertion.

The next proposition is used in our proof of Propositions 6.13 and 6.14. In the forthcoming article Nag2, this proposition is also used to investigate the boundary divisors $D_{1}$ and $D_{2}$ of triplets $\left(V, D_{1} \cup D_{2}, U\right)$.

Proposition 6.7. Suppose that the assumptions in Proposition 6.6 hold. Let $\pi: \widetilde{S}_{2} \rightarrow S_{2}$ be the minimal resolution. Then $B_{0}(F)=1, B_{1}(F)=0$ and $B_{2}(F)=B_{2}\left(S_{2}\right)+\sharp\left(C \cap S_{1}\right)+2 p_{a}(C)-1$.

Proof. Since $F$ is the support of a member of $\left|-K_{S_{2}}\right|$ and $-K_{S_{2}}$ is ample, we have $B_{0}(F)=1$. Applying Lemma 2.4 by setting $X=\mathbb{P}^{3} \backslash S_{1}, S=S_{2} \backslash F$ and $r=C \backslash\left(C \cap S_{1}\right)$, we have the following by Proposition 6.6.

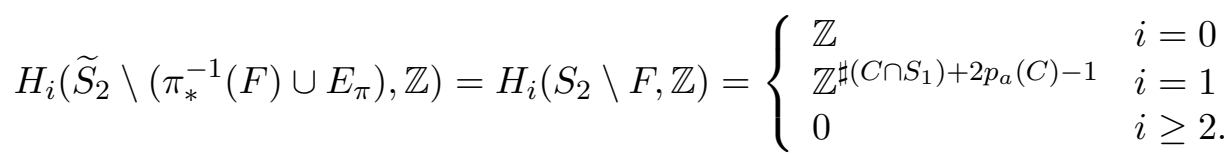

Now we consider the following exact sequence of cohomologies:

$$
\begin{array}{r}
\cdots \longrightarrow H^{1}\left(\widetilde{S}_{2}, \mathbb{Z}\right) \rightarrow H^{1}\left(\pi_{*}^{-1}(F) \cup E_{\pi}, \mathbb{Z}\right) \\
\rightarrow H^{2}\left(\widetilde{S}_{2}, \pi_{*}^{-1}(F) \cup E_{\pi}, \mathbb{Z}\right) \rightarrow H^{2}\left(\widetilde{S}_{2}, \mathbb{Z}\right) \rightarrow H^{2}\left(\pi_{*}^{-1}(F) \cup E_{\pi}, \mathbb{Z}\right) \\
\rightarrow H^{3}\left(\widetilde{S}_{2}, \pi_{*}^{-1}(F) \cup E_{\pi}, \mathbb{Z}\right) \rightarrow H^{3}\left(\widetilde{S}_{2}, \mathbb{Z}\right) \longrightarrow \cdots .
\end{array}
$$


By the Lefschetz duality, we have $H^{i}\left(\widetilde{S}_{2}, \pi_{*}^{-1}(F) \cup E_{\pi}, \mathbb{Z}\right) \cong H_{4-i}\left(\widetilde{S}_{2} \backslash\right.$ $\left.\left(\pi_{*}^{-1}(F) \cup E_{\pi}\right), \mathbb{Z}\right)$. We also note that $H^{1}\left(\widetilde{S}_{2}, \mathbb{Z}\right)=H^{3}\left(\widetilde{S}_{2}, \mathbb{Z}\right)=0$ since $S_{2}$ is rational. Hence $B_{1}\left(\pi_{*}^{-1}(F) \cup E_{\pi}, \mathbb{Z}\right)=0$ and $B_{2}\left(\pi_{*}^{-1}(F) \cup E_{\pi}\right)=$ $B_{2}\left(\widetilde{S}_{2}\right)+\sharp\left(C \cap S_{1}\right)+2 p_{a}(C)-1$. Moreover, we have the following exact sequence of cohomologies by Lemma 2.5.

$$
\begin{aligned}
\cdots & \rightarrow H^{1}\left(S_{2}, \mathbb{Z}\right) \rightarrow H^{1}(F, \mathbb{Z}) \oplus H^{1}\left(\widetilde{S}_{2}, \mathbb{Z}\right) \rightarrow H^{1}\left(\pi_{*}^{-1}(F) \cup E_{\pi}, \mathbb{Z}\right) \\
& \rightarrow H^{2}\left(S_{2}, \mathbb{Z}\right) \rightarrow H^{2}(F, \mathbb{Z}) \oplus H^{2}\left(\widetilde{S}_{2}, \mathbb{Z}\right) \rightarrow H^{2}\left(\pi_{*}^{-1}(F) \cup E_{\pi}, \mathbb{Z}\right) \\
& \rightarrow H^{3}\left(S_{2}, \mathbb{Z}\right) .
\end{aligned}
$$

We note that $H^{1}\left(S_{2}, \mathbb{Z}\right)=H^{3}\left(S_{2}, \mathbb{Z}\right)=0$ since $S_{2}$ is rational. Hence $B_{1}(F)=0$ and

$B_{2}(F)=B_{2}\left(S_{2}\right)+B_{2}\left(\pi_{*}^{-1}(F) \cup E_{\pi}\right)-B_{2}\left(\widetilde{S}_{2}\right)=B_{2}\left(S_{2}\right)+\sharp(C \cup F)+2 p_{a}(C)-1$.

6.3. The case $W_{1}=\mathbb{P}^{3}$. In this subsection, we assume that $W_{1}=\mathbb{P}^{3}$. By (6.0.1), the surface $S_{1}$ (resp. $S_{2}$ ) is a hyperplane which does not contain $C$ (resp. a cubic hypersurface which contains $C$ such that $C \not \subset \operatorname{Sing} S_{2}$ ).

Proposition 6.8. It holds that $p_{a}(C) \leq 1$.

Proof. As $B_{3}(V)=2 p_{a}(C)$, it holds that

(6.3.1) $4 \geq \chi_{\mathrm{top}}(F)=\chi_{\mathrm{top}}\left(S_{2}\right)+2 p_{a}(C)+N_{1}+N_{2}-N_{1 \cap 2}-2 \geq 2 p_{a}(C)$

by Lemma 6.3, Proposition 6.2 and Theorem 2.1. Hence $2 \geq p_{a}(C)$.

Suppose that $p_{a}(C)=2$. Since all equalities of (6.3.1) hold, we have $\chi_{\text {top }}\left(S_{2}\right)=1$ and hence $S_{2}$ is a cone over an elliptic curve by Theorem 2.1.

By [H-W81, its minimal resolution $\widetilde{S}_{2}$ is a geometrically ruled surface over the elliptic curve, which corresponds to a vector bundle of degree 3 . We write $\tau_{*}^{-1}(C) \equiv a C_{0}+b f$, where $C_{0}$ (resp. $f$ ) is the minimal section (resp. a ruling) of $\widetilde{S}_{2}$. Then we have

$$
\begin{aligned}
2=2 p_{a}\left(\tau_{*}^{-1}(C)\right)-2 & =\left(\tau_{*}^{-1}(C) \cdot \tau_{*}^{-1}(C)+K_{\widetilde{S}_{2}}\right) \\
& =\left(a C_{0}+b f \cdot(a-2) C_{0}+(b-3) f\right) \\
& =(2 b-3 a)(a-1)
\end{aligned}
$$

by the genus formula. Hence $(a, b)=(2,4)$ or $(3,5)$, which implies that $\tau_{*}^{-1}(C)$ is reducible. This contradicts the irreducibility of $C$.

By [M-M81, Table 2] and Proposition 6.8, $C$ is either a smooth rational curve of degree $1 \leq d \leq 4$ or an elliptic curve of degree $3 \leq d \leq 5$. The following completes the proof of Theorem 1.1 (1) in this case.

Proposition 6.9. The center of the blow-up $C$ cannot be an elliptic curve of degree 5 .

Proof. Suppose that $C$ is an elliptic curve of degree 5. By Lemma 6.3, Proposition 6.2 and Theorem 2.1, we have

$$
4 \geq \chi_{\text {top }}(F) \geq \chi_{\text {top }}\left(S_{2}\right)+1 \geq 2 .
$$


Suppose that $S_{2}$ is not a cone. Then all equalities of (6.3.2) hold by Theorem [2.1. Hence $\chi_{\text {top }}\left(S_{2}\right)=3$ and $F$ is a sum of three concurrent lines on $S_{1} \cong \mathbb{P}^{2}$. If $S_{2}$ is normal, then each sum of three $(-1)$-curves is not concurrent by Qia02, Appendix: Configurations of the Singularity types], a contradiction. If $S_{2}$ is non-normal, then $S_{2}$ belongs to the class (C) of A-F83 and the conductor locus $\bar{E}_{S_{2}}$ of the normalization of $S_{2}$ is reducible, which contradicts Lemma 2.3 .

Hence $S_{2}$ must be a cone. By [H-W81] and [A-F83, the resolution $\tau: \widetilde{S}_{2} \rightarrow S_{2}$ contracts the minimal section $C_{0}$ of the geometrically ruled surface $\widetilde{S}_{2}$, which corresponds to a vector bundle of degree 3 on a curve. We also note that $\tau^{*}\left(-K_{S_{2}}\right) \sim C_{0}+3 f$, where $f$ is a ruling of $\widetilde{S}_{2}$. We can write $\tau_{*}^{-1}(C) \sim a C_{0}+b f$ with $3 b \geq a>0$ since $C$ is not a ruling of $S_{2}$. Then we have

$$
5=\left(C \cdot-K_{S_{2}}\right)_{S_{2}}=\left(\tau_{*}^{-1}(C) \cdot \tau^{*}\left(-K_{S_{2}}\right)\right)_{\widetilde{S}_{2}}=\left(a C_{0}+b f \cdot C_{0}+3 f\right)_{\widetilde{S}_{2}}=b .
$$

This implies $\tau_{*}^{-1}(C) \sim C_{0}+5 f$. Hence $C$ must be singular since $\left(\tau_{*}^{-1}(C)\right.$. $\left.C_{0}\right)=2$, which contradicts the smoothness of $C$.

6.4. The case $W_{1}=\mathbb{Q}^{3}$. In this subsection, we assume that $W_{1}=\mathbb{Q}^{3}$. By (6.0.1), the surface $S_{1}$ (resp. $S_{2}$ ) is a hyperplane section which does not contain $C$ (resp. a member of $\left|\mathcal{O}_{\mathbb{Q}^{3}}(2)\right|$ which contains $C$ such that $C \not \subset$ Sing $S_{2}$ ).

Proposition 6.10. It holds that $p_{a}(C) \leq 1$.

Proof. Note that $\chi_{\text {top }}\left(S_{1}\right) \geq 3$ since $S_{1}$ is either $\mathbb{P}^{1} \times \mathbb{P}^{1}$ or $\mathbb{Q}_{0}^{2}$. Since $B_{3}\left(\mathbb{Q}^{3}\right)=0$, we obtain the desired inequality

$$
5 \geq \chi_{\mathrm{top}}(F) \geq \chi_{\mathrm{top}}\left(S_{1}\right)+\chi_{\mathrm{top}}\left(S_{2}\right)+2 p_{a}(C)-4 \geq 2 p_{a}(C)+2
$$

by Theorem 2.1, Lemma 2.2, Proposition 6.2 and Lemma 6.3,

Lemma 6.11. If $S_{1} \cong \mathbb{P}^{1} \times \mathbb{P}^{1}$, then we have $\chi_{\text {top }}(F) \leq 4$.

Proof. Suppose that $B_{2}(F)=4$. By the proof of Lemma 6.3, it suffices to show that $B_{1}(F)>0$. Since $F$ is the support of a member of $\left|\mathcal{O}_{\mathbb{P}^{1} \times \mathbb{P}^{1}}(2,2)\right|$, $F$ consists of four rulings $f_{i j}$ for $1 \leq i, j \leq 2$ with $\left(f_{k j} \cdot f_{l j}\right)=\delta_{k l}$. Hence $B_{1}(F)=1$.

By [M-M81, Table 2] and Proposition 6.10, $C$ is either a smooth rational curve of degree $1 \leq d \leq 4$ or an elliptic curve of degree $4 \leq d \leq 5$. To prove Theorem 1.1 (1), we have only to show the following:

Proposition 6.12. The center of the blow-up $C$ cannot be an elliptic curve of degree 5 .

Proof. Suppose that $C$ is an elliptic curve of degree 5. Then $V$ is of No.17 in [M-M81, Table 2] and hence given by the blow-up $\varphi_{2}: V \rightarrow \mathbb{P}^{3}$ of $\mathbb{P}^{3}$ along an elliptic curve of degree 5 (see [Mat95, $\S$ III-3]). Applying Proposition 6.9 by exchanging the subscripts of $\varphi_{1}$ and $\varphi_{2}$, we have the assertion.

Proposition 6.13. The center of the blow-up $C$ cannot be an elliptic curve of degree 4 . 
Proof. Suppose that $C$ is an elliptic curve of degree 4. Since $C$ is a complete intersection of divisors in $\left|\mathcal{O}_{\mathbb{Q}^{3}}(1)\right|$ and $\left|\mathcal{O}_{\mathbb{Q}^{3}}(2)\right|$, the surface $S_{2}$ is normal. Also, $S_{2}$ is rational by [H-W81] and Lemma 2.2. By the same method as in Proposition 6.10, we obtain

$$
5 \geq \chi_{\text {top }}(F) \geq \chi_{\text {top }}\left(S_{1}\right)+\chi_{\text {top }}\left(S_{2}\right)+2 p_{a}(C)-4 \geq \chi_{\text {top }}\left(S_{1}\right)+1 .
$$

Therefore $S_{1}$ cannot be $\mathbb{P}^{1} \times \mathbb{P}^{1}$ by Lemma 6.11. Hence $S_{1} \cong \mathbb{Q}_{0}^{2}$ and $\mathbb{Q}^{3} \backslash S_{1} \cong \mathbb{A}^{3}$ by [Fur93, Theorem A]. We can rewrite the above inequality as

$$
5 \geq \chi_{\mathrm{top}}(F) \geq \chi_{\mathrm{top}}\left(S_{2}\right)+2 p_{a}(C)-1=\chi_{\mathrm{top}}\left(S_{2}\right)+1 \geq 4 .
$$

By Proposition 6.7, one of the following two cases occurs:

(1) $B_{1}(F)=0, B_{2}(F)=4, B_{2}\left(S_{2}\right) \leq 2$ or

(2) $B_{1}(F)=0, B_{2}(F)=3, B_{2}\left(S_{2}\right)=1$.

For each case, the intersection $F$ is a sum of concurrent lines since $\mathbb{Q}_{0}^{2}$ contains $F$. However, each sum of four $(-1)$-curves in $S_{2}$ is not concurrent when $B_{2}\left(S_{2}\right) \leq 2$ and there is at most two $(-1)$-curves in $S_{2}$ when $B_{2}\left(S_{2}\right)=$ 1 by Qia02, Appendix: Configurations of the Singularity types]. Hence we have a contradiction to the existence of $F$.

6.5. The case $W_{1}=V_{5}$. In this subsection, we assume that $W_{1}=V_{5}$. By (6.0.1), the surface $S_{1}$ (resp. $S_{2}$ ) is a hyperplane section which does not contain $C$ (resp. a hyperplane section which contains $C$ such that $C \not \subset \operatorname{Sing} S_{2}$ ). By [M-M81, Table 2], $C$ is either a smooth rational curve of degree $1 \leq d \leq 3$ or a complete intersection of two hyperplane sections. To prove Theorem 1.1 (1), we have only to show the following:

Proposition 6.14. The center of the blow-up $C$ cannot be a complete intersection of two hyperplane sections.

Proof. Suppose that $C$ is a complete intersection of two hyperplane sections. Then the surface $S_{2}$ is normal and rational by Lemma 2.2] and [H-W81]. By Proposition 6.2 and Lemma 6.3, we have

$$
\begin{aligned}
6 \geq 1+B_{2}(F) & \geq \chi_{\mathrm{top}}\left(S_{1}\right)+\chi_{\mathrm{top}}\left(S_{2}\right)+N_{1}+N_{2}-N_{1 \cap 2}-3 \\
& \geq \chi_{\mathrm{top}}\left(S_{1}\right)+\chi_{\mathrm{top}}\left(S_{2}\right)-2 .
\end{aligned}
$$

Claim 2. It holds that $\chi_{\text {top }}\left(S_{1}\right)=3$.

Proof of Claim 2. By Theorem 2.1 (4) and Lemma 2.2, we have $\chi_{\text {top }}\left(S_{i}\right) \geq 3$ for $i=1,2$. Suppose that $\chi_{\text {top }}\left(S_{1}\right) \geq 4$. By (6.5.1), we have $B_{2}(F) \geq 4$ and hence $F \subset S_{2}$ has at least three lines. By Qia02, Appendix: Configurations of the Singularity types], the surface $S_{2}$ contains only one line if $\chi_{\text {top }}\left(S_{2}\right)=3$. Hence $\chi_{\text {top }}\left(S_{2}\right) \geq 4$. By (6.5.1), we have $\chi_{\text {top }}\left(S_{2}\right)=4$ and $F$ is a sum of 5 lines. This contradicts the fact that $S_{2}$ contains at most three lines by Qia02, Appendix: Configurations of the Singularity types].

Hence $S_{1}$ is either normal with an $A_{4}$-singularity which contains only one $(-1)$-curve by Qia02, Appendix: Configurations of the Singularity types] or non-normal belonging to the class (C) of [A-F83. Hence $V_{5} \backslash S_{1} \cong \mathbb{A}^{3}$ by [Fur93, Theorem A]. By Proposition 6.7, one of the following three cases occurs:

(1) $B_{2}(F)=5$ 
(2) $B_{2}(F)=4, B_{2}\left(S_{2}\right) \leq 2$,

(3) $B_{2}(F)=3, B_{1}(F)=0, B_{2}\left(S_{2}\right)=1$

If the case (1) occurs, then $F$ is a sum of 5 lines. However, $S_{1}$ cannot contain such $F$ by Qia02, Appendix: Configurations of the Singularity types] and Lemma 2.3, a contradiction.

If the case (2) occurs, then $F$ contains 3 lines. Hence $S_{1}$ must be nonnormal and $S_{2}$ contains at most three lines. Hence $F \subset S_{1}$ contains a conic, but $S_{1}$ contains no conic (see [Kis05, Lemma 4.5, 4.6]), a contradiction.

Hence the case (3) must occur. Since $S_{2}$ contains the unique (-1)-curve, $F$ is a sum of the line and two conics. In particular, the intersection $S_{1} \cap S_{2}$ is reduced. Since the line contains an $A_{4}$-singular point $p_{1} \in S_{2}$, we obtain $p_{1} \in \operatorname{Sing} F$.

Note that $N_{1}+N_{2}-N_{1 \cap 2}=1$ by (6.5.1) and hence $N_{1}=\sharp(C \cap F)=1$ by Proposition 6.2 (2). Let $p_{2} \in C \cap F$ be the point. Since $C$ is an ample divisor on $S_{2}$, all irreducible component of $F$ must contain $p_{2}$. Since $B_{1}(F)=0$, the point $p_{2}$ is the only concurrent point on $F$ and hence we have $p_{1}=p_{2}$. Then the smooth Cartier divisor $C$ on $S_{2}$ contains a singular point $p_{1}$, which gives a contradiction.

\section{ExClusion of PRIMitive FAno 3-FoldS}

The aim of this section is to prove Theorem 1.1 (1) when $V$ is primitive. Hence it suffices to show that the following cases cannot occur (see Mat95, $\S[I I-3]):$

- $V$ is a Fano 3-fold of No.2. It is given by a double covering $g: V \rightarrow$ $\mathbb{P}^{1} \times \mathbb{P}^{2}$ whose branch locus $B$ is a divisor of bidegree $(2,4)$. One extremal contraction $\varphi_{1}=\operatorname{pr}_{1} \circ g$ of $V$ is of $D_{1}$-type and the other $\varphi_{2}=\mathrm{pr}_{2} \circ g$ is of $C_{1}$-type. We also have $B_{3}(V)=40$.

- $V$ is a Fano 3-fold of No.6. Its extremal contractions $\varphi_{1}$ and $\varphi_{2}$ are $C_{1}$-type of discriminant degree 6 . We also have $B_{3}(V)=18$.

- $V$ is a Fano 3-fold of No.8. It is given by a double covering $g: V \rightarrow$ $V_{7}:=\mathbb{P}_{\mathbb{P}^{2}}(\mathcal{O} \oplus \mathcal{O}(1))$ whose branch locus is a member of $\left|-K_{V_{7}}\right|$. Note that $V_{7}$ has extremal contractions $\psi_{1}: V_{7} \rightarrow \mathbb{P}^{3}$ and $\psi_{2}: V_{7} \rightarrow \mathbb{P}^{2}$. One extremal contraction $\varphi_{1}$ of $V$ is the Stein factorization of $\psi_{1} \circ g$, which is of $E_{3}$ or $E_{4}$-type, and the other $\varphi_{2}$ is the composite $\psi_{2} \circ g$, which is of $C_{1}$-type. We also have $B_{3}(V)=18$.

- $V$ is a Fano 3-fold of No.18. It is given by a double covering $g: V \rightarrow$ $\mathbb{P}^{1} \times \mathbb{P}^{2}$ whose branch locus $B$ is a divisor of bidegree $(2,2)$. One extremal contraction $\varphi_{1}=\operatorname{pr}_{1} \circ g$ of $V$ is of $D_{2}$-type, and the other $\varphi_{2}=\mathrm{pr}_{2} \circ g$ is of $C_{1}$-type. We also have $B_{3}(V)=4$.

- $V$ is a Fano 3 -fold of No.32. It is a divisor on $\mathbb{P}^{2} \times \mathbb{P}^{2}$ of bidegree $(1,1)$.

- $V$ is a Fano 3 -fold of No.35. It is the blow-up of $\mathbb{P}^{3}$ at a point.

In what follows, we exclude the possibilities of above six cases separately.

Proposition 7.1. The Fano 3-fold $V$ cannot be of either No.32 or No.35.

Proof. Each of Fano 3-folds of No.32 and No.35 has different extremal contractions of length two. Hence we have the assertion by Proposition 3.4 (2). 
Proposition 7.2. The Fano 3-fold $V$ cannot be of No.2.

Proof. Suppose that $V$ is a Fano 3-fold of No.2. We may assume that $D_{1} \sim$ $H_{1}$ and $D_{2} \sim H_{2}$ by Proposition 3.4 .

Let us compute topological Euler characteristics of boundaries. As $g\left(D_{1}\right) \cong$ $\mathbb{P}^{2}$ and $\left.B\right|_{g\left(D_{1}\right)} \sim \mathcal{O}_{\mathbb{P}^{2}}(4)$, it holds that

$$
\chi_{\mathrm{top}}\left(D_{1}\right)=2 \chi_{\mathrm{top}}\left(g\left(D_{1}\right)\right)-\chi_{\mathrm{top}}\left(\left.B\right|_{g\left(D_{1}\right)}\right) \geq 6-5=1 .
$$

As $g\left(D_{2}\right) \cong \mathbb{P}^{1} \times \mathbb{P}^{1}$ and $\left.B\right|_{g\left(D_{2}\right)} \sim \mathcal{O}_{\mathbb{P}^{1} \times \mathbb{P}^{1}}(2,4)$, it holds that

$$
\chi_{\text {top }}\left(D_{2}\right)=2 \chi_{\text {top }}\left(g\left(D_{2}\right)\right)-\chi_{\text {top }}\left(\left.B\right|_{g\left(D_{2}\right)}\right) \geq 8-(1+2+4)=1 .
$$

As $-K_{V}$ is ample, it holds that

$$
\chi_{\text {top }}\left(D_{1} \cap D_{2}\right) \leq B_{0}\left(D_{1} \cap D_{2}\right)+B_{2}\left(D_{1} \cap D_{2}\right) \leq 2\left(D_{1} \cdot D_{2} \cdot-K_{V}\right)=4 .
$$

Hence we obtain the following inequality which contradicts Lemma 3.5.

$$
\chi_{\text {top }}\left(D_{1} \cap D_{2}\right) \leq 4<37 \leq \chi_{\text {top }}\left(D_{1}\right)+\chi_{\text {top }}\left(D_{2}\right)+B_{3}(V)-5 .
$$

Proposition 7.3. The Fano 3-fold $V$ cannot be of No.6.

Proof. Suppose that $V$ is a Fano 3-fold of No.6. We may assume that $D_{1} \sim$ $H_{1}$ and $D_{2} \sim H_{2}$ by Proposition 3.4.

Let us compute topological Euler characteristics of boundaries. Fix $i \in$ $\{1,2\}$. Then $\left.\varphi_{i}\right|_{D_{i}}$ is a conic bundle. If the image of $\left.\varphi_{i}\right|_{D_{i}}$ is not contained in the discriminant locus, then the general fiber of $\left.\varphi_{i}\right|_{D_{i}}$ is smooth and we have

$$
\chi_{\text {top }}\left(D_{i}\right) \geq \chi_{\text {top }}\left(\mathbb{P}^{1}\right) \times \chi_{\text {top }}\left(\mathbb{P}^{1}\right)=4 .
$$

Otherwise, the general fiber of $\left.\varphi_{i}\right|_{D_{i}}$ is a reducible conic and the special fiber is a non-reduced line. Since $\left.\varphi_{i}\right|_{D_{i}}$ has at most $6-1=5$ special fiber, we have

$$
\chi_{\text {top }}\left(D_{i}\right) \geq 3 \times\left(\chi_{\text {top }}\left(\mathbb{P}^{1}\right)-5\right)+2 \times 5=1 .
$$

Since $-K_{V}$ is ample, we have

$$
\chi_{\text {top }}\left(D_{1} \cap D_{2}\right) \leq B_{0}\left(D_{1} \cap D_{2}\right)+B_{2}\left(D_{1} \cap D_{2}\right) \leq 2\left(D_{1} \cdot D_{2} \cdot-K_{V}\right)=8 .
$$

Hence we obtain the following inequality which contradicts Lemma 3.5.

$$
\chi_{\text {top }}\left(D_{1} \cap D_{2}\right) \leq 8<15 \leq \chi_{\text {top }}\left(D_{1}\right)+\chi_{\text {top }}\left(D_{2}\right)+B_{3}(V)-5 .
$$

Proposition 7.4. The Fano 3-fold $V$ cannot be of No.8.

Proof. Suppose that $V$ is a Fano 3-fold of No.8. We may assume that $D_{1} \sim$ $H_{1}$ and $D_{2} \sim H_{2}$ by Proposition 3.4.

Let us compute topological Euler characteristics of the boundary divisors. As $g\left(D_{1}\right)=\mathbb{P}^{2} \sim \psi_{1}^{*} \mathcal{O}_{\mathbb{P}^{3}}(1)$ and $\left.B\right|_{g\left(D_{1}\right)} \sim \mathcal{O}_{\mathbb{P}^{2}}(4)$, it holds that

$$
\chi_{\text {top }}\left(D_{1}\right)=2 \chi_{\text {top }}\left(\mathbb{P}^{2}\right)-\chi_{\text {top }}\left(\left.B\right|_{g\left(D_{1}\right)}\right) \geq 6-5=1 \text {. }
$$

As $g\left(D_{2}\right)=\mathbb{F}_{1}$ and $\left.B\right|_{g\left(D_{2}\right)} \sim 2 \Sigma_{1}+4 f_{1}$, it holds that

$$
\chi_{\text {top }}\left(D_{2}\right)=2 \chi_{\text {top }}\left(\mathbb{F}_{1}\right)-\chi_{\text {top }}\left(\left.B\right|_{g\left(D_{2}\right)}\right) \geq 8-6=2 \text {. }
$$


As $g\left(D_{1}\right) \cap g\left(D_{2}\right) \cong \mathbb{P}^{1}$, it holds that

$$
\chi_{\text {top }}\left(D_{1} \cap D_{2}\right)=2 \chi_{\text {top }}\left(\mathbb{P}^{1}\right)-\chi_{\text {top }}\left(g\left(D_{1}\right) \cap g\left(D_{2}\right) \cap B\right) \leq 4 .
$$

Hence we obtain the following inequality which contradicts Lemma 3.5.

$$
\chi_{\text {top }}\left(D_{1} \cap D_{2}\right) \leq 4<16 \leq \chi_{\text {top }}\left(D_{1}\right)+\chi_{\text {top }}\left(D_{2}\right)+B_{3}(V)-5 .
$$

Proposition 7.5. The Fano 3-fold V cannot be of No.18.

Proof. Suppose that $V$ is a Fano 3-fold of No.18. Since the length of $\varphi_{2}$ is one, we may assume that $D_{1} \sim H_{1}+H_{2}$ and $D_{2} \sim H_{2}$ by Proposition 3.4.

Let us compute topological Euler characteristics of $D_{2}$ and $D_{1} \cap D_{2}$. Since $-\left.K_{D_{2}} \sim\left(H_{1}+H_{2}\right)\right|_{D_{2}}$, the surface $D_{2}$ is a Gorenstein del Pezzo surface of degree $\left(-K_{D_{2}}\right)^{2}=4$. As both $H_{1}+H_{2}$ and $\left.D_{1}\right|_{D_{2}} \sim-K_{D_{2}}$ are ample, we have

$$
\chi_{\text {top }}\left(D_{1} \cap D_{2}\right) \leq 1+B_{2}\left(D_{1} \cap D_{2}\right) \leq 1+\left(D_{1} \cdot D_{2} \cdot H_{1}+H_{2}\right)=5 .
$$

Claim 3. It holds that $\chi_{\text {top }}\left(D_{2}\right) \geq 4$. Moreover, $\chi_{\text {top }}\left(D_{2}\right)=4$ implies that $\chi_{\text {top }}\left(D_{1} \cap D_{2}\right) \leq 4$.

Proof of Claim 3. Suppose that $D_{2}$ is normal. Then the general fiber of the conic bundle $\left.\varphi_{2}\right|_{D_{2}}$ is smooth. Hence $\chi_{\text {top }}\left(D_{2}\right) \geq \chi_{\text {top }}\left(\mathbb{P}^{1}\right) \times \chi_{\text {top }}\left(\mathbb{P}^{1}\right)=4$.

Suppose that $D_{2}$ is non-normal. Let $\sigma_{D_{2}}: \bar{D}_{2} \rightarrow D_{2}$ be the normalization. Then $D_{2}$ is not a cone by Lemma 2.2 and hence $D_{2}$ belongs to

$$
\begin{cases}\text { the class (B) of } \mathrm{A}-\mathrm{F} 83 & \text { if } \bar{D}_{2} \cong \mathbb{P}^{2}, \\ \text { the class (C) of } \mathrm{A}-\mathrm{F} 83 & \text { if } \bar{D}_{2} \cong \mathbb{F}_{2} \text { or } \\ \text { the class (D) of } \mathrm{A}-\mathrm{F} 83 & \text { if } \bar{D}_{2} \cong \mathbb{F}_{0} .\end{cases}
$$

We note that $\sigma_{D_{2}}^{*}\left(\left.H_{i}\right|_{D_{2}}\right)$ is a nef and effective divisor in $\bar{D}_{2}$ with selfintersection number 0 for $i=1,2$. As $\left(H_{1} \cdot H_{2} \cdot D_{2}\right)=1$, we have $\sigma_{D_{2}}^{*}\left(\left.H_{1}\right|_{D_{2}}\right) \nsim$ $\sigma_{D_{2}}^{*}\left(\left.H_{2}\right|_{D_{2}}\right)$. Hence only the case that $\bar{D}_{2} \cong \mathbb{F}_{0}$ can occur and $D_{2}$ belongs to the class (D). By [A-F83, $\sigma_{D_{2}}$ is an isomorphism without one ruling $\Sigma_{0}$ and $\left.\sigma_{D_{2}}\right|_{\Sigma_{0}}$ is a double covering to $\mathbb{P}^{1}$. Hence $\chi_{\text {top }}\left(D_{2}\right)=4$ and we have the first assertion.

We prove the second assertion below. To seek a contradiction, we assume that $\chi_{\text {top }}\left(D_{2}\right)=4$ and $\chi_{\text {top }}\left(D_{1} \cap D_{2}\right)=5$. Then $B_{1}\left(D_{1} \cap D_{2}\right)=0$ and $D_{1} \cap D_{2}$ consists of four curves whose intersection number with $\left.\left(H_{1}+H_{2}\right)\right|_{D_{2}} \sim-K_{D_{2}}$ is one by (7.0.1).

Suppose that $D_{2}$ is normal. Then $\left.D_{1}\right|_{D_{2}} \subset D_{2}$ is a sum of four (-1)-curves with trivial first Betti number. However, Qia02, Appendix: Configurations of the Singularity types] shows us that every sum of four (-1)-curves in $D_{2}$ has non-trivial first Betti number, a contradiction.

Suppose that $D_{2}$ is non-normal. Then we have shown that $D_{2}$ belongs to the class (D). By [A-F83, we have $\sigma_{D_{2}}^{*}\left(-K_{D_{2}}\right) \sim \Sigma_{0}+2 f_{0}$. Then $\sigma_{D_{2 *}}{ }^{-1}\left(\left.D_{1}\right|_{D_{2}}\right)$ consists of four curves whose intersection number with $\Sigma_{0}+$ $2 f_{0}$ is one. Hence each of four curves is linearly equivalent to $f_{0}$. However, this implies that $\left.D_{1}\right|_{D_{2}}$ is disconnected since $\sigma_{D_{2}}$ is as stated above, which contradicts the ampleness of $\left.D_{1}\right|_{D_{2}}$. 
By Lemma 3.5 and Claim 3, we have

$$
\chi_{\mathrm{top}}\left(D_{1}\right)=\chi_{\mathrm{top}}\left(D_{1} \cap D_{2}\right)-\chi_{\mathrm{top}}\left(D_{2}\right)-B_{3}(V)+5 \leq 1,
$$

but we obtain $\chi_{\text {top }}\left(D_{1}\right) \geq 2$ by Lemmas 7.6 7.11 below, a contradiction.

Here we explain how to deduce $\chi_{\text {top }}\left(D_{1}\right) \geq 2$ from Lemmas 7.6 7.11, If it holds that $\chi_{\text {top }}\left(D_{1}\right) \leq 3$, then $D_{1}$ is a certain non-normal surface (Lemma (7.6). Suppose $D_{1}$ is such a certain non-normal surface. Then its normalization is a $\mathbb{P}^{1}$-bundle over a smooth curve (Lemma 7.7) and the arithmetic genus $g_{a}$ of the curve is either 0 or 1 (Lemmas 7.8 7.9). We can compute $\chi_{\text {top }}\left(D_{1}\right) \geq 3$ when $g_{a}=0$ (Lemma 7.10) and $\chi_{\text {top }}\left(D_{1}\right)=2$ when $g_{a}=1$ (Lemma 7.11). Hence the inequality $\chi_{\text {top }}\left(D_{1}\right) \geq 2$ always holds.

Lemma 7.6. If $\chi_{\text {top }}\left(D_{1}\right) \leq 3$, then $D_{1}$ is non-normal with $\left(E_{D_{1}} \cdot H_{1}\right)>0$, where $E_{D_{1}}$ is the conductor locus of $D_{1}$.

Proof. As $D_{1} \sim H_{1}+H_{2}$ and $\varphi_{1}$ is a conic bundle, so is $\left.\varphi_{1}\right|_{D_{1}}$. Suppose that $D_{1}$ is normal. Then a general fiber of $\left.\varphi_{1}\right|_{D_{1}}$ is smooth and hence $\chi_{\text {top }}\left(D_{1}\right) \geq \chi_{\text {top }}\left(\mathbb{P}^{1}\right) \times \chi_{\text {top }}\left(\mathbb{P}^{1}\right)=4$. The same conclusion holds when $D_{1}$ is non-normal with $\left(E_{D_{1}} \cdot H_{1}\right)=0$.

Lemma 7.7. Suppose that $D_{1}$ is non-normal with $\left(E_{D_{1}} \cdot H_{1}\right)>0$. Let $\sigma: \bar{D}_{1} \rightarrow D_{1}$ be the normalization and $\bar{E}_{D_{1}} \subset \bar{D}_{1}$ the conductor locus of $\bar{D}_{1}$. Then $\bar{D}_{1}$ is a $\mathbb{P}^{1}$-bundle over a smooth curve $Z$.

Proof. Let $\pi: \widetilde{D}_{1} \rightarrow \bar{D}_{1}$ be the minimal resolution and $\tau:=\sigma \circ \pi$. Let $A$ be an effective divisor on $\widetilde{D}_{1}$ such that $K_{\widetilde{D}_{1}} \sim \pi^{*}\left(K_{\bar{D}_{1}}\right)-A$. Then we have

$\tau^{*}\left(\left.\left(H_{1}+H_{2}\right)\right|_{D_{1}}\right)+K_{\widetilde{D}_{1}} \sim \tau^{*}\left(\left.H_{1}\right|_{D_{1}}-K_{D_{1}}\right)+K_{\widetilde{D}_{1}} \sim \tau^{*}\left(\left.H_{1}\right|_{D_{1}}\right)-A-\pi^{*} \bar{E}_{D_{1}}$.

As $\left(E_{D_{1}} \cdot H_{1}\right)>0$, it holds that

$$
\begin{aligned}
& \left(\tau^{*}\left(\left.\left(H_{1}+H_{2}\right)\right|_{D_{1}}\right)+K_{\widetilde{D}_{1}} \cdot \tau^{*}\left(\left.H_{1}\right|_{D_{1}}\right)\right)_{\widetilde{D}_{1}} \\
= & \left(\tau^{*}\left(\left.H_{1}\right|_{D_{1}}\right)-A-\pi^{*} \bar{E}_{D_{1}} \cdot \tau^{*}\left(\left.H_{1}\right|_{D_{1}}\right)\right)_{\widetilde{D}_{1}} \\
\leq & -\left(\left.\pi^{*} \bar{E}_{D_{1}} \cdot \tau^{*} H_{1}\right|_{D_{1}}\right)_{\widetilde{D}_{1}}=-\left(\left.\bar{E}_{D_{1}} \cdot \sigma^{*} H_{1}\right|_{D_{1}}\right)_{\bar{D}_{1}}<0 .
\end{aligned}
$$

Hence $\tau^{*}\left(\left.\left(H_{1}+H_{2}\right)\right|_{D_{1}}\right)+K_{\widetilde{D}_{1}}$ is not nef and there is an extremal ray $R \subset \overline{\mathrm{NE}}\left(\widetilde{D}_{1} / \mathbb{P}^{1}\right)$ with respect to $\varphi_{1} \circ \tau$ such that

$$
\left(\tau^{*}\left(\left.\left(H_{1}+H_{2}\right)\right|_{D_{1}}\right)+K_{\widetilde{D}_{1}} \cdot R\right)_{\widetilde{D}_{1}}<0 .
$$

Let $g: \widetilde{D}_{1} \rightarrow Z$ be an extremal contraction which corresponds to $R$. Since $\varphi_{1} \circ \tau: \widetilde{D}_{2} \rightarrow \mathbb{P}^{1}$ is dominant, we have $\operatorname{dim} Z=1$ or 2 .

Suppose that $\operatorname{dim} Z=2$. Then there is a curve $C \subset \widetilde{D}_{1}$ such that $C^{2}<0$ and $\left(C \cdot K_{\widetilde{D}_{1}}\right)_{\widetilde{D}_{1}}<-\left(C \cdot \tau^{*}\left(\left.\left(H_{1}+H_{2}\right)\right|_{D_{1}}\right)\right)_{\widetilde{D}_{1}} \leq 0$. Hence $C$ is a $(-1)$ curve such that $\left(C \cdot \tau^{*}\left(\left.\left(H_{1}+H_{2}\right)\right|_{D_{1}}\right)\right)_{\widetilde{D}_{1}}=\left(\pi_{*}(C) \cdot \sigma^{*}\left(\left.\left(H_{1}+H_{2}\right)\right|_{D_{1}}\right)\right)_{\bar{D}_{1}}=$ 0 . Since $\sigma^{*}\left(\left.\left(H_{1}+H_{2}\right)\right|_{D_{1}}\right)$ is ample, the morphism $\sigma$ contracts $C$, which contradicts the minimality of $\sigma$.

Hence $\operatorname{dim} Z=1$ and $g: \widetilde{D}_{1} \rightarrow Z$ is a $\mathbb{P}^{1}$-bundle. Let $f$ be a ruling of $\widetilde{D}_{1}$. Then we have

$$
-2=\left(f \cdot K_{\widetilde{D}_{1}}\right)_{\widetilde{D}_{1}}<-\left(f \cdot \tau^{*}\left(\left.\left(H_{1}+H_{2}\right)\right|_{D_{1}}\right)\right)_{\widetilde{D}_{1}} \leq 0 .
$$


As $f^{2}=0$, the morphism $\pi$ does not contract $f$. We note that $\tau^{*}\left(\left.H_{2}\right|_{D_{1}}\right)$ is a sum of rulings. Hence

$$
\begin{aligned}
& \left(f \cdot-\tau^{*} K_{D_{1}}\right)_{\widetilde{D}_{1}}=\left(f \cdot \tau^{*}\left(\left.\left(H_{1}+H_{2}\right)\right|_{D_{1}}\right)\right)_{\widetilde{D}_{1}}=1 \text { and } \\
& \left(f \cdot A+\pi^{*} \bar{E}_{D_{1}}\right)_{\widetilde{D}_{1}}=-\left(f \cdot \tau^{*}\left(\left.\left(H_{1}+H_{2}\right)\right|_{D_{1}}\right)+K_{\widetilde{D}_{1}}\right)_{\widetilde{D}_{1}}=1 .
\end{aligned}
$$

Since $\left(\tau^{*}\left(\left.H_{1}\right|_{D_{1}}\right) \cdot \pi^{*} \bar{E}_{D_{1}}\right)_{\widetilde{D}_{1}}>0$, it holds that

$$
\left(f \cdot \pi^{*} \bar{E}_{D_{1}}\right)_{\widetilde{D}_{1}}=1 \text { and }(f \cdot A)_{\widetilde{D}_{1}}=0 .
$$

Then $A$ is a sum of rulings. Hence $A=0$ and $\pi$ is crepant. As $g: \widetilde{D}_{1} \rightarrow Z$ is $\mathbb{P}^{1}$-bundle, the surface $\bar{D}_{1}$ is smooth unless $\bar{D}_{1} \cong \mathbb{Q}_{0}^{2}$ and $\widetilde{D}_{1} \cong \mathbb{F}_{2}$. However, $\bar{D}_{1} \neq \mathbb{Q}_{0}^{2}$ because $\varphi_{1} \circ \sigma: \bar{D}_{1} \rightarrow \mathbb{P}^{1}$ is dominant. Hence $\pi$ is the identity and we have the assertion by regarding $g$ as a morphism from $\bar{D}_{1}$.

Lemma 7.8. Let $S$ be a non-normal weak del Pezzo surface and $\sigma: \bar{S} \rightarrow S$ the normalization of $S$. Let $E$ (resp. $\bar{E}$ ) be the conductor locus of $S$ (resp. $\bar{S}$ ). Then $\operatorname{dim} H^{0}\left(\mathcal{O}_{\bar{E}}\right)=1$. Moreover, one of the following two cases occurs:

(1) $\operatorname{dim} H^{1}\left(\mathcal{O}_{\bar{E}}\right)=0,\left(\sigma^{*} K_{S} \cdot \bar{E}\right)_{\bar{S}}=-2,\left(K_{S} \cdot E\right)_{S}=-1$

(2) $\operatorname{dim} H^{1}\left(\mathcal{O}_{\bar{E}}\right)=1,\left(\sigma^{*} K_{S} \cdot \bar{E}\right)_{\bar{S}}=0,\left(K_{S} \cdot E\right)_{S}=0$

Proof. This follows from the same argument as in the proof of Mor82, Lemma 3.35].

Lemma 7.9. Under the assumptions of Lemma 7.7, it holds that $\left(f \cdot \bar{E}_{D_{1}}\right)_{\bar{D}_{1}}$ $=-\left(f \cdot \sigma^{*} K_{D_{1}}\right)_{\bar{D}_{1}}=1, \sigma^{*}\left(\left.H_{1}\right|_{D_{1}}\right) \equiv 2 f$ and $g_{a}(Z)=g_{a}\left(\bar{E}_{D_{2}}\right) \leq 1$,

Proof. The first assertion holds by (7.0.2) and (7.0.3). As $-\left(\sigma^{*}\left(\left.H_{1}\right|_{D_{1}}\right)\right.$. $\left.\sigma^{*} K_{D_{1}}\right)_{\bar{D}_{1}}=\left(H_{1} \cdot H_{2} \cdot D_{1}\right)=2$, we have the second assertion.

Since $-\left.K_{D_{1}} \sim H_{2}\right|_{D_{1}}$ is nef and big, the surface $D_{1}$ is a Gorenstein weak del Pezzo surface. Hence the last assertion holds by Lemma 7.8 and the genus formula.

Lemma 7.10. If $Z$ is rational, then $\chi_{\mathrm{top}}\left(D_{1}\right) \geq 3$.

Proof. By Lemmas 7.7 and 7.9, the surface $\bar{D}_{1}$ is a Hirzebruch surface which contains a section $-\sigma^{*} K_{D_{1}}$ whose self-intersection number is 2 . Since $p_{a}\left(\bar{E}_{D_{1}}\right)=0$, we have $-\left(\sigma^{*} K_{D_{1}} \cdot E_{D_{1}}\right)_{\bar{D}_{1}}=2$ by Lemma 7.8, Hence one of the following two cases occurs:

(1) $\bar{D}_{1} \cong \mathbb{F}_{0}$ and $\sigma^{*} K_{D_{1}} \sim E_{D_{1}} \sim \Sigma_{0}+f_{0}$.

(2) $\bar{D}_{1} \cong \mathbb{F}_{2}$ and $\sigma^{*} K_{D_{1}} \sim E_{D_{1}} \sim \Sigma_{2}+2 f_{2}$.

Suppose that the case (1) occurs. Then $D_{1}$ is a non-normal del Pezzo surface of class $(\mathrm{C})$ in $\mathrm{A}-\mathrm{F} 83$, which implies $E_{D_{1}} \cong \mathbb{P}^{1}$. Hence $\chi_{\text {top }}\left(D_{1}\right)=$ $\chi_{\text {top }}\left(\bar{D}_{1}\right)-\chi_{\text {top }}\left(\bar{E}_{D_{1}}\right)+\chi_{\text {top }}\left(E_{D_{1}}\right) \geq 3$.

Suppose that the case (2) occurs. Then we have

$$
\left(\bar{E}_{D_{1}} \cdot \sigma^{*}\left(\left.H_{1}\right|_{D_{1}}\right)\right)_{\bar{D}_{1}}=\left(\bar{E}_{D_{1}} \cdot \sigma^{*}\left(\left.H_{2}\right|_{D_{1}}\right)\right)_{\bar{D}_{1}}=2 \text {. }
$$

Suppose that $\bar{E}_{D_{1}}$ is irreducible. Then so is $E_{D_{1}}$. Since $\left.\sigma\right|_{E_{D_{1}}}$ is not birational, we have $\left(E_{D_{1}} \cdot H_{1}\right)=1$ by (7.0.4). Since $\left.\varphi_{1}\right|_{E_{D_{1}}}: E_{D_{1}} \rightarrow \mathbb{P}^{1}$ is birational, we obtain $E_{D_{1}} \cong \mathbb{P}^{1}$ and $\chi_{\text {top }}\left(D_{1}\right)=\chi_{\text {top }}\left(\bar{D}_{1}\right)-\chi_{\text {top }}\left(\bar{E}_{D_{1}}\right)+$ $\chi_{\text {top }}\left(E_{D_{1}}\right)=4$. 
Suppose that $\bar{E}_{D_{1}}$ is reducible. Then $\bar{E}_{D_{1}}$ consists of rulings $C_{1}, C_{2}$, and $\Sigma_{2}$. Since $\left(\Sigma_{2} \cdot \sigma^{*}\left(\left.H_{2}\right|_{D_{1}}\right)\right)_{\bar{D}_{1}}=0$, the curve $\sigma\left(\Sigma_{2}\right)$ is an irreducible component of a fiber of the conic bundle $\varphi_{2}$ and hence $\sigma\left(\Sigma_{2}\right) \cong \mathbb{P}^{1}$.

Fix $i \in 1,2$. Since $\left(C_{i} \cdot \sigma^{*}\left(\left.H_{1}\right|_{D_{1}}\right)\right)_{\bar{D}_{1}}=0$, we have $\sigma\left(\Sigma_{2}\right) \neq \sigma\left(C_{i}\right)$ and $\sigma\left(C_{i}\right)$ is an irreducible component of an intersection $\left.D_{1}\right|_{H_{1}}$. Since $H_{1}$ is isomorphic to a quadric surface in $\mathbb{P}^{3}$ and $\left.D_{1}\right|_{H_{1}} \sim-\frac{1}{2} K_{H_{1}}$, we have $\sigma\left(C_{i}\right) \cong$ $\mathbb{P}^{1}$. As $\left(C_{i} \cdot \sigma^{*}\left(\left.H_{2}\right|_{D_{1}}\right)\right)_{\bar{D}_{1}}=1$, the morphism $\left.\sigma\right|_{C_{i}}$ is an isomorphism. Hence $\sigma\left(C_{1}\right)=\sigma\left(C_{2}\right)$ since both of them are contained in $E_{D_{1}}$.

Since $\left(\sigma\left(C_{1}\right) \cdot H_{2}\right)=1,\left(\sigma\left(\Sigma_{2}\right) \cdot H_{2}\right)=0$ and $E_{D_{1}}$ is connected, we have $\sharp\left(\sigma\left(\Sigma_{2}\right) \cap \sigma\left(C_{1}\right)\right)=1$. Hence $\chi_{\text {top }}\left(D_{1}\right)=\chi_{\text {top }}\left(\bar{D}_{1}\right)-\chi_{\text {top }}\left(\bar{E}_{D_{1}}\right)+$ $\chi_{\text {top }}\left(E_{D_{1}}\right) \geq 4-4+3=3$.

Lemma 7.11. If $Z$ is elliptic, then $\chi_{\text {top }}\left(D_{1}\right)=2$.

Proof. The surface $\bar{D}_{1}$ is isomorophic to $\mathbb{P}_{Z}(\mathcal{E})$ for some normalized vector bundle $\mathcal{E}$ of rank two. Let $C_{0}$ be a minimal section of $\bar{D}_{1}$ and $e:=\operatorname{deg}(\operatorname{det} \mathcal{E})$. By Lemmas 7.8 and 7.9, we can write $\bar{E}_{D_{1}} \equiv C_{0}+$ af and $-\sigma^{*}\left(K_{D_{1}}\right) \equiv C_{0}+$ $(e-a) f$ for $a \in \mathbb{Z}$. Since $-e+2(e-a)=\left(-\sigma^{*}\left(K_{D_{1}}\right)\right)_{D_{1}}^{2}=\left(H_{2}^{2} \cdot\left(H_{1}+H_{2}\right)\right)=$ 2, we obtain $\bar{E}_{D_{1}} \equiv C_{0}+\left(\frac{e}{2}-1\right) f$ and $-\sigma^{*}\left(K_{D_{1}}\right) \equiv C_{0}+\left(\frac{e}{2}+1\right) f$.

By [Har77, Theorem V.2.15], we have $e \geq-1$. Since $0 \leq\left(C_{0} \cdot \sigma^{*}\left(\left.H_{2}\right|_{D_{1}}\right)\right)_{\bar{D}_{1}}$ $=1-\frac{e}{2}$, we have $e=0,2$. If $e=0$, then $C_{0}$ is nef and $\left(\bar{E}_{D_{1}} \cdot C_{0}\right)_{\bar{D}_{1}}=-1$, a contradiction. Hence $e=2$ and $\bar{E}_{D_{1}}=C_{0}$.

As $\left(\bar{E}_{D_{1}} \cdot \sigma^{*}\left(\left.H_{2}\right|_{D_{1}}\right)\right)_{D_{1}}=0$, the curve $E_{D_{1}}$ is an irreducible component of a conic. Hence $E_{D_{1}} \cong \mathbb{P}^{1}$ and $\chi_{\text {top }}\left(D_{1}\right)=\chi_{\text {top }}\left(\bar{D}_{1}\right)-\chi_{\text {top }}\left(\bar{E}_{D_{1}}\right)+$ $\chi_{\mathrm{top}}\left(E_{D_{1}}\right)=2$.

Summarizing the arguments in $₫ 6$ and $\$ 7$, we complete the proof of Theorem $1.1(1)$.

\section{REFERENCES}

[A-F83] M. Abe, M. Furushima. On non-normal del Pezzo surfaces. Math. Nachrichten, 260, 3-13 (2003)

[Bri68] E. Brieksorn. Rationale Singularitäten komplexer Flächen. Invent. Math. 4, 336358 (1968)

[B-D89] D. Brenton, D. Drucker. Perfect graphs and complex surface singularities with perfect local fundamental group, Tohoku Math. J. 41, 507-525 (1989)

[Fuj82] T. Fujita. On the topology of non-complete algebraic surfaces. J. Fac. Sci. Univ. Tokyo, Sect. IA 29, 503-566 (1982)

[Fuj90] T. Fujita. On singular del Pezzo varieties. Algebraic geometry (L'Aquila, 1988), 117-128, Lecture Notes in Math. 1417, Springer, Berlin, (1990).

[Fur86] M. Furushima. Singular del Pezzo surfaces and analytic compactifications of 3dimensional complex affine space $\mathbb{C}^{3}$. Nagoya Math. J. 104, 1-28 (1986)

[Fur90] M. Furushima. Complex analytic compactifications of $\mathbb{C}^{3}$. Compos. Math. 76, 163196 (1990)

[Fur92] M. Furushima. A new example of a compactification of $\mathbb{C}^{3}$. Math. Z. 212, 395-399 (1993)

[Fur93] M. Furushima. The complete classification of compactifications of $\mathbb{C}^{3}$ which are projective manifolds with the second Betti number one. Math. Ann. 297, 627-662 (1993)

[Fur00] M. Furushima. A birational construction of projective compactifications of $\mathbb{C}^{3}$ with second Betti number equal to one. Ann. Mat. Pura Appll. (4) 178, 115-128 (2000) 
[F-N89a] M. Furushima, N. Nakayama. A new construction of a compactification of $\mathbb{C}^{3}$. Tohoku Math. J. 41, 543-560 (1989)

[F-N89b] M. Furushima, N. Nakayama. The family of lines on the Fano threefold $V_{5}$. Nagoya Math. J. 116, 111-122 (1989)

[Har77] R. Hartshorne. Algebraic geometry. Graduate Texts in Mathematics, No. 52. Springer-Verlag, New York-Heidelberg (1977)

[Ham83] Helmut A. Hamm. Zur Homotopietyp Steinscher Räume (German) [On the homotopy type of Stein spaces]. J. Reine Angew. Math. 338, 121-135 (1983)

[Hir54] F. Hirzebruch. Some problems on differentiable and complex manifolds. Ann. of Math. (2) 60, 213-236 (1954)

[H-W81] F. Hidaka, K. Watanabe. Normal Gorenstein surfaces with ample anti-canonical divisor. Tokyo J. Math. 4, 319-330 (1981)

[Ish14] S. Ishii. Introduction to singularities, Springer, Tokyo (2014)

[Isk80] V.A. Iskovskih. Anticanonical models of three-dimensional algebraic varieties, J. Soviet Math. 13-14, 745-814 (1980)

[Kal02] Sh. Kaliman. Polynomials with general $\mathbb{C}^{2}$-fibers are variables. Pacific Journal of Math. 203, No.1, 161-190 (2002)

[K-Z99] Sh. Kaliman. Zaidenberg, M.: Affine modifications and affine hypersurfaces with a very transitive automorphism groups. Transformation Groups 4, 53-95 (1999)

[Kis05] T. Kishimoto. Compactifications of contractible affine 3-folds into smooth Fano 3-folds with $B_{2}=2$. Math. Z. 251, 783-820 (2005)

[KPR89] H. Kraft, T. Petrie, J. D. Randall. Quotient varieties. Adv. Math. 74, no. 2, 145$162(1989)$

[Mat95] K. Matsuki. Weyl groups and birational transformations among minimal models. Mem. Amer. Math. Soc. 116, no. 557 (1995)

[Miy78] M. Miyanishi. Lectures on curves on rational and unirational surfaces. Tata Inst. Fund. Res. Bombay (1978)

[Mor82] S. Mori. Threefolds whose canonical bundles are not numerically effective. Ann. of Math. 115, 133-176 (1982)

[M-M81] S. Mori, S. Mukai. Classification of Fano 3-folds with $B_{2} \geq 2$. Manuscripta Math 36, 147-162 (1981)

[M-M83] S. Mori, S. Mukai. On Fano 3-folds with $B_{2} \geq 2$. Adv. Stud. Pure Math. 1, 101$129(1983)$

[Nag2] M. Nagaoka. Fano compactifications of contractible affine 3-folds with trivial log canonical divisors (II). In preparation.

[Pet89] Th. Peternell. Compactifications of $\mathbb{C}^{3}$. II. Math. Ann. 283, no. 1, 121-137 (1989)

[P-S88] Th. Peternell, M. Schneider. Compactifications of $\mathbb{C}^{3}$. I. Math. Ann. 280, no. 1, 129-146 (1988)

[Qia02] Y. Qiang. On Gorenstein log del Pezzo surfaces. Jpn. J. of Math. 28, 87-136 (2002)

Graduate School of Mathematical Sciences, The University of Tokyo, 38-1 Komaba, Meguro-Ku, TOKyo 153-8914, Japan

E-mail address: nagaoka@ms.u-tokyo.ac.jp 